Research Article

\title{
Combined Extracts of Epimedii Folium and Ligustri Lucidi Fructus with Budesonide Attenuate Airway Remodeling in the Asthmatic Rats by Regulating Apoptosis and Autophagy
}

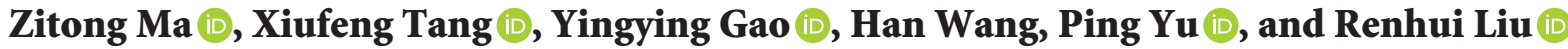 \\ School of Traditional Chinese Medicine, Capital Medical University, No. 10 Xitoutiao, Youanmenwai, Fengtai District, \\ Beijing 100069, China \\ Correspondence should be addressed to Renhui Liu; gzblrh45@ccmu.edu.cn
}

Received 24 April 2020; Revised 6 July 2020; Accepted 7 July 2020; Published 5 August 2020

Academic Editor: Teh Ley Kek

Copyright (c) 2020 Zitong Ma et al. This is an open access article distributed under the Creative Commons Attribution License, which permits unrestricted use, distribution, and reproduction in any medium, provided the original work is properly cited.

\begin{abstract}
This study aimed to investigate the effects of the coadministration of budesonide (Bud) and the extracts of Epimedii Folium and Ligustri Lucidi Fructus (EEL) on regulating apoptosis and autophagy in asthmatic rats. Forty Sprague-Dawley rats were divided randomly into five groups (8 rats in each group): normal control (control), asthma model (asthma), Bud (1 mg Bud suspension in $50 \mathrm{ml}$ sterile physiological saline for $30 \mathrm{~min}$ ), EEL (100 mg/kg EEL), and group of coadministration of Bud and EEL (Bud\&EEL, $100 \mathrm{mg} / \mathrm{kg}$ EEL plus Bud by nebulized inhalation for $30 \mathrm{~min}$ ). Rats were sensitized and challenged with ovalbumin for 7 weeks and treated with corresponding drug for 4 weeks. We anesthetized all rats with $25 \%$ ethyl carbamate $(4 \mathrm{ml} / \mathrm{kg})$ and took lung tissues and BALF after final ovalbumin challenge to observe the lung histopathology and morphometry; apoptosis in BALF and lung tissue; protein expressions of Ki-67, $\alpha$-SMA, cleaved Caspase-3, p-mTOR, and LC3; and protein and mRNA expressions of Bax, Bcl-2, Caspase-3, P53, mTOR, and Beclin-1. Results showed that Bud\&EEL could alleviate airway remodeling, inhibit cell proliferation and autophagy in lung tissue, and promote apoptosis in BALF and lung tissue in ovalbumin-induced asthma rats through downregulating the protein expressions of $\alpha$-SMA and Ki-67, the protein ratio of LC3-II/LC3-I and Bcl-2/Bax, and the protein and mRNA expressions of Bcl-2 and Beclin-1, while upregulating the protein expressions of cleaved Caspase- 3 and p-mTOR, and the protein and mRNA expressions of Bax, Caspase-3, P53, and mTOR. Bud\&EEL had better effects than single-use Bud on improving airway remodeling, promoting apoptosis, and regulating the expressions of autophagy- and apoptosis-related proteins. This study suggested that the effects of coadministration of EEL and Bud on regulating apoptosis and autophagy were better than those of single-use Bud treatment, and that might be the mechanism of attenuating airway remodeling, providing an alternative therapy for asthma.
\end{abstract}

\section{Introduction}

Asthma is one of the most serious chronic respiratory diseases characterized by airway inflammation, airway remodeling, and bronchial hyperresponsiveness. It affects all age brackets $[1,2]$, and its morbidity and mortality are increasing year by year worldwide [3]. Airway remodeling-regarded as a primary cause of refractory asthma-is characterized by epithelial shedding, goblet cell hyperplasia, basal membrane thickening, airway smooth muscle cell hyperplasia and hypertrophy, and bronchial vasculature angiogenesis [4]. It occurs in all-age asthmatic patients and can exacerbate airway inflammation and bronchial hyperresponsiveness [5]. However, researchers have not clarified the mechanism of airway remodeling and have not found the targeted treatment for it at present. Glucocorticoids (GCs), especially inhaled GCs, became the first-line treatment for asthma because of the prominent anti-inflammatory effect and rapid onset of action [6]. Inhaled GCs at conventional doses play small roles in severe asthma $[7,8]$, while administration at high doses or in a long term will induce a series of side effects such as growth stunting in children, GC dependent even resistance, and hypothalamicpituitary-adrenal (HPA) axis dysfunction [9-11]. Improving therapeutic and side effects of GCs through drug combination has a great significance for the treatment of asthma. 
Apoptosis and autophagy are important physiological processes to maintain cellular environmental homeostasis, participating in regulated cell death (RCD) [12]. Dysregulated RCD is related to various kinds of diseases such as cancer, autoimmune disease, and Alzheimer's disease [13]. However, RCD is especially important for lung which is exposed to the environment (pathogens and aerosolized toxins) and needs to maintain the network of epithelialendothelial interfaces to ensure gas exchange [12]. Dysfunctions of apoptosis and autophagy in asthma have been validated in both of animal experiments and clinical studies. The activity of apoptosis in the airway biopsy of subjects with severe asthma is greater than that in normal subjects [14]. Airway inflammation is one of the most important pathological features of asthma; resolution of inflammation is regulated by production of local autacoid, apoptosis of inflammatory cells, and phagocytosis of surrounding phagocytes [15]. Reducing eosinophil apoptosis can aggravate the grade of asthma, and accumulation of eosinophils can result in airway inflammation, organ dysfunction, and airway remodeling [16]. Current and potential antiasthmatic drugs can all have effect on the number of eosinophils. Besides, in some of them, it has been verified that this effect is related to regulating eosinophils apoptosis, such as glucocorticoids, theophylline, and anti-Siglec-F [16-18]. Autophagy is a highly conserved cellular degradation process in all eukaryotes; it can degrade damaged proteins and organelle (e.g., mitochondria) to promote cellular turnover, involved in many diseases such as lung disease, heart disease, and cancer [19]. In asthma, autophagy is implicated in immune responses. It participates in regulating cell differentiation of plasmocyte, development and survival of lymphocyte, and maintaining immunological memory of B cells $[20,21]$. The activity of autophagy in sputum granulocytes, peripheral blood cells, and peripheral blood eosinophils rises abnormally in severe asthmatic patients [22]. Single nucleotide polymorphism (SNP) analyses show that the variants of autophagy-related gene (ATG) allele are associated with childhood asthma and the expression of ATG5 mRNA is increased significantly in acute asthma [23]. In addition, inhibiting autophagy can improve the airway hyperresponsiveness and inflammation in ovalbumin-induced asthmatic rats $[21,24]$. The interaction between apoptosis and autophagy is worth noting: autophagy-related proteins interact with apoptosis-related proteins and some proteins can have effects on both apoptosis and autophagy $[25,26]$. In addition, this interaction plays a key role in airway remodeling through maintaining cellular homeostasis and mediating cell death process [27], indicating that the dysregulated apoptosis and autophagy are involved in asthmatic pathogenesis [28, 29]. This means that the regulation of apoptosis and autophagy may be one of mechanisms of improving airway remodeling in asthma.

Compounds derived from Traditional Chinese Medicine (TCM) are essential sources of seeking drugs which can improve the side and therapeutic effects of hormonotherapy. The combination of Epimedii Folium (EF) and Ligustri Lucidi Fructus (LLF) is a traditional formula and is widely used in treating asthma (also called wheeze syndrome in
TCM), achieving excellent curative effect. The extracts of EF and LLF (EEL) are mainly icariin and oleanolic acid. EF and LLF are used widely in the treatment of osteoporosis, cancer, sexual dysfunction, and asthma and have the functions of increasing bone mineral density and adjusting sex hormone, in addition to their antioxidative and anti-inflammatory effects [30-32]. There are also many studies which show the effects of EF and LLF on apoptosis and autophagy. Both of $\mathrm{EF}$ and LLF can regulate apoptosis by affecting Caspase-3 [33]. Icariin, an active ingredient of EF, suppresses oxygenglucose deprivation and reperfusion-induced apoptosis and autophagy in PC12 cells [34]. Oleanolic acid, an active ingredient of LLF, has anticancer effects by regulating intracellular environment: it can promote apoptosis of HL60 cells through death-receptor pathway [35] and induce autophagy of bladder cancer cells through AMPK-mTOR-ULK1 signaling pathway [36].

In previous studies, we found that the coadministration of EEL and GCs (dexamethasone and budesonide) had better effects on airway remodeling and inflammation by reducing the levels of interleukin-4 (IL-4), IL-5, and IgE in serum and inhibiting the TGF- $\beta 1 /$ Smad pathway in ovalbumin-induced asthmatic rats compared with single-use GC treatment [37, 38]; EEL can increase the sensitivity of asthmatic rats to budesonide by promoting lymphocyte apoptosis and balancing GR/HSP90 [39]. We speculate that the coadministration of EEL and GC could act synergistically to restore the dysregulated apoptosis and autophagy involved in asthmatic pathogenesis based on the interaction between apoptosis and autophagy. In the present study, we selected ovalbumin-induced asthma rats to explore and compare the effects of EEL and budesonide separately or combinedly in apoptosis and autophagy.

\section{Methods}

2.1. Preparation of Active Ingredients. Epimedii Folium (EF, dried leaf of Epimedium brevicornum Maxim) and Ligustri Lucidi Fructus (LLF, dried mature seed of Ligustrum lucidum Ait.) were purchased from Beijing Tongrentang pharmaceutical Co. Ltd., China. They were authenticated by an expert herbalist, Shiyuan Jin, Honorary Professor, School of TCM, Capital Medical University. Voucher specimens were deposited at the TCM Endocrine and Metabolic Disease Laboratory of TCM School of Capital Medical University, China. Preparation of active ingredients of EF and LLF, including flavonoids and iridoids, was performed according to the methods described before [40]. And the process of extracting combined active ingredients has been protected by the Chinese patent 20140037992.5. The active ingredients of EF and LLF were mixed at a ratio of 2 to 3, equivalent to the raw herbal ratio of 4 to 3 according to clinical practice. Before application, the combined active fractions were dissolved with distilled water at $10 \mathrm{mg} / \mathrm{ml}$.

2.2. Animals. 40 male Sprague-Dawley rats, weighing 120 to $130 \mathrm{~g}$, were purchased from Vital River Laboratory Animal Technology Co. Ltd. (Beijing, China). he experiment 
complied with the Animal Management Rule of Ministry of Public THealth, China, and the experimental protocol was approved by Animal Care Committee of Capital Medical University, Beijing, China. All the animals were cared for in Experimental Animal Center of Capital Medical University. During the whole experiment, the animals were housed in stainless cages (three rats per cage) at conventional controlled conditions (temperature of $23 \pm 2^{\circ} \mathrm{C}$, relative humidity of $50 \pm 10 \%$, and 12-hour light-dark cycle). They were allowed free access to the standard laboratory food and tap water.

2.3. Experimental Protocol. After acclimatization for 7 days, the rats were randomly assigned to 5 groups ( $n=8$ per group): normal control group (control), asthma model group (asthma), budesonide group (Bud), group of extracts of EF and LLF (EEL), and group of coadministration of Bud and EEL (Bud\&EEL). The experimental protocols were performed according to our previous study [39], as explained in Scheme 1. All rats except rats in control group were sensitized with $1 \mathrm{mg}$ ovalbumin (Grade II, Sigma-Aldrich, St. Louis, MO, USA) and $100 \mu \mathrm{g}$ aluminum hydroxide (Sigma) in $1 \mathrm{ml}$ sterile physiological saline by intraperitoneal injection (i.p. $0.5 \mathrm{ml}$ ) and subcutaneous injection (s.c.; 5 spots: hind feet on both sides, groins on both sides, and back; every spot was injected with $0.1 \mathrm{ml}$.) on the $1 \mathrm{st}$ and 8th days and challenged with $1 \mathrm{mg}$ ovalbumin in $100 \mathrm{ml}$ sterile physiological saline by nebulized inhalation with a flow of $0.8 \mathrm{ml} / \mathrm{min}$ for $30 \mathrm{~min}$ for 7 weeks. Rats in control group were treated with sterile physiological saline. On the 35th to 64th days, rats were administrated with corresponding drug once a day. Control and asthma groups were treated with isometric distilled water; Bud group was treated with Bud (1 mg Bud suspension in $50 \mathrm{ml}$ sterile physiological saline, AstraZeneca Pty Ltd., New South Wales, Australia) by nebulized inhalation with a flow of $1.6 \mathrm{ml} / \mathrm{min}$ for $30 \mathrm{~min}$; EEL group was treated with EEL $(100 \mathrm{mg} / \mathrm{kg}$ body weight) by gavage; Bud\&EEL group was treated with both of Bud and EEL. On the 65th day, we anesthetized all rats with $25 \%$ ethyl carbamate ( $4 \mathrm{ml} / \mathrm{kg}$ body weight, i.p.), opened rat's chest, and took out lung tissue along with trachea.

2.4. Lung Histopathology and Morphometry. The middle lobe of the left lung was cut off and fixed by $4 \%$ paraformaldehyde, for preparation to be embedded by paraffin, and then routinely processed. Lung tissue sections were stained with hematoxylin and eosin (H\&E), periodic acid-Schiff (PAS), and Masson's trichrome and then measured with the Nikon ECLIPSE 80i biomicroscope and NIS-Elements BR 3.2 image analysis system (Nikon, Japanese) according to our previous study [38].

We surveyed the perimeter of basement membrane $(\mathrm{Pbm})$, total area of bronchus (Wat1), area of lumen (Wat2), area of outer margin of the smooth muscle (Wam1), and area of medial smooth muscle (Wam2) in H\&E-stained sections; then calculated the standardized thickness of airway wall $(\mathrm{Wat}, \mathrm{Wat}=(\mathrm{Wat} 1-\mathrm{Wat} 2) / \mathrm{Pbm})$ and the standardized thickness of airway smooth muscle (Wam, $\mathrm{Wam}=(\mathrm{Wam} 1-\mathrm{Wam} 2) / \mathrm{Pbm})$; observed the goblet cells and $\mathrm{Pbm}$ in PAS-stained sections; then calculated the standardized number (number/Pbm) and area (area/Pbm) of PAS-positive goblet cells; determined collagen fiber area (stained in blue) and $\mathrm{Pbm}$ in Masson's trichrome-stained lung sections; and then calculated the mean score of the fibrotic area divided by $\mathrm{Pbm}$ in each rat.

2.5. TdT-Mediated dUTP Nick-End Labeling (TUNEL) Assay for Apoptosis in BALF and Lung Tissue. The cell-debris pellets of bronchoalveolar lavage fluid (BALF) samples and lung tissue (paraffin sections) were prepared to determine apoptosis activity using the TUNEL assay (Beyotime Co., Ltd., Shanghai, China) according to manufacturer's instructions.

2.6. Immunofluorescence Analysis (IF). We mainly used paraffin-embedded lung sections for immunofluorescent staining. The paraffin sections were dewaxed with xylene, ethanol, and double distilled water, antigen-retrieved with citrate buffer using microwave oven, and blocked with $10 \%$ normal goat serum for 2 hours. Then, they were incubated with primary antibodies against alpha smooth muscle actin ( $\alpha$-SMA), Ki-67, Bcl-2, Bax, Caspase-3, P53, mTOR, and Beclin-1, respectively, overnight at $4^{\circ} \mathrm{C}$. On the second day, the sections were incubated with relevant fluorescent secondary antibodies (Keygen Biotech Co., Ltd., Jiangsu, China) at $37^{\circ} \mathrm{C}$ for $2 \mathrm{~h}$ and mounted with antifade mounting medium containing DAPI (Solarbio Science \& Technology Co., Ltd., Beijing, China). At least 3 views with airway were analyzed per rat in fluorescence microscope. All measurements were performed with the Nikon ECLIPSE $80 \mathrm{i}$ biomicroscope and NIS-Elements BR 3.2 image analysis system (Nikon, Japanese). The description and concentration of primary antibodies used in IF analysis are listed in Table 1.

2.7. Western Blot Analysis (WB). The total protein was extracted from $60 \mathrm{mg}$ lung tissue using RIPA lysis buffer. Concentration of the protein was quantified by quantitative bromochloroacetic acid (BCA) protein kit (Beijing Biosynthesis Biotechnology Co., Ltd., Beijing, China). The protein was mixed with $4 \times$ SDS-PAGE loading buffer (dilution rate $3: 1$ ) and boiled at $100^{\circ} \mathrm{C}$ for $10 \mathrm{~min}$ to make it denatured. Equal amounts of protein $(30 \mu \mathrm{g}$ per lane) were separated by sodium dodecyl sulfate polyacrylamide gel electrophoresis (SDS-PAGE) and transferred into $0.45 \mu \mathrm{m}$ polyvinylidene fluoride (PVDF) membranes. After blocking with 5\% nonfat-dried milk at room temperature for $2 \mathrm{~h}$, membranes were incubated with primary antibodies overnight at $4^{\circ} \mathrm{C}$. Membranes were detected with relevant horseradish peroxidase-labeled secondary antibodies (Zhong Shan Golden Bridge Biotechnology Co., Ltd., Beijing, China; $1: 20000$ ) for $1 \mathrm{~h}$. The protein band was visualized by an electrochemiluminescent (ECL) reagent and exposed to X-film. GAPDH or $\beta$-actin was used for normalization. The mean density of each protein band was measured by ImageJ software (National Institutes of Health, USA). The description and concentration of primary antibodies used in WB analysis are listed in Table 2. 


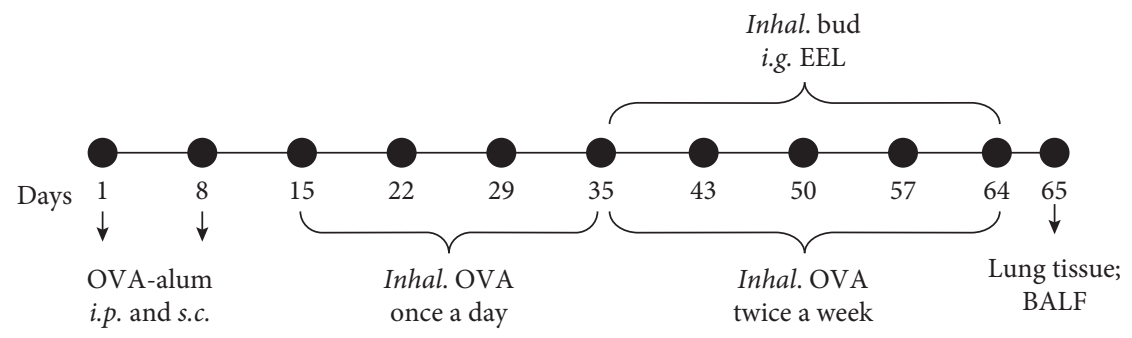

Scheme 1: The experimental protocol. Following the sensitization and challenges of ovalbumin, rats were treated with Bud, EEL, or distilled water on days 35-64 and then sacrificed with acquisition of lung tissue and BALF on day 65. OVA-alum: ovalbumin dissolved in aluminum hydroxide adjuvant; i.p.: intraperitoneal injection; s.c.: subcutaneous injection; Inhal.: nebulized inhalation; i.g.: gastric perfusion.

TABLe 1: Antibodies used for IF analysis.

\begin{tabular}{|c|c|c|c|}
\hline Antibody & Description & Concentration & Manufacturer \\
\hline$\alpha$-SMA & Mouse & $1: 200$ & Bioss, Beijing, China \\
\hline Ki-67 & Rabbit & $1: 250$ & Abcam, Cambridge, UK \\
\hline Bcl-2 & Mouse & $1: 200$ & Santa Cruz Biotechnology, Santa Cruz, USA \\
\hline Bax & Rabbit & $1: 100$ & Abcam, Cambridge, UK \\
\hline Caspase-3 & Rabbit & $1: 200$ & Abcam, Cambridge, UK \\
\hline P53 & Mouse & $1: 200$ & Santa Cruz Biotechnology, Santa Cruz, USA \\
\hline mTOR & Rabbit & $1: 100$ & Abcam, Cambridge, UK \\
\hline Beclin-1 & Rabbit & $1: 200$ & Abcam, Cambridge, UK \\
\hline
\end{tabular}

2.8. Quantitative Real-Time PCR Analysis (qPCR). Total RNA was isolated from the lung tissue with the RNAprep pure Tissue Kit (TianGen Co., Ltd., Beijing, China) according to the manufacturer's recommendations. Total RNA $(2 \mu \mathrm{g})$ was reverse-transcribed using the FastKing RT Kit (with gDNase) (TianGen Co., Ltd., Beijing, China) to generate complementary DNA (cDNA). SuperReal PreMix Plus (SYBR-green) and Bio-Rad PCR cycler (CFX96 RealTime System) were used for real-time quantitative analysis. The PCR program was performed for 40 cycles with each cycle consisting of $12 \mathrm{~s}$ of denaturation at $95^{\circ} \mathrm{C}, 1 \mathrm{~min}$ of annealing at $60^{\circ} \mathrm{C}$, and $10 \mathrm{~s}$ of extension at $60^{\circ} \mathrm{C}$. The mRNA expression was quantified as previously described [41]. The primers used in the qPCR analysis are presented in Table 3.

2.9. Statistical Analysis. Results of all measurements were presented as means \pm standard deviation (SD). The statistical differences $(P<0.05)$ among experimental groups were evaluated using SPSS 21.0 software (SPSS Inc., Chicago, USA) by one-way analysis of variance (ANOVA). The least significant difference (LSD) test when the variances were equal or Tamhane's T2 test when the variances were not equal was used for comparisons between individual groups and to determine which means differed statistically significantly $(P<0.05)$.

\section{Results}

3.1. Effects of Bud and EEL on Airway Remodeling. We observed the Pbm, Wat, and Wam to assess the features of inflammatory cell infiltration and airway remodeling in H\&Estained lung sections. As shown in Figure 1(g), no inflammation, mucosal edema, or epithelial lesions were observed in the control group. However, severe inflammation, mucosal edema, epithelial lesions, and inflammatory cell infiltration including lymphocyte and eosinophil were observed in asthma model group which were attenuated by administration of Bud, EEL, or Bud\&EEL. Wat and Wam in the asthma group were greater than those in the control group (Figures 1(b) and 1(c); all $P<0.01$ ). Compared with asthma group, Wat was remarkably decreased in EEL and Bud\&EEL groups $(P<0.05$ or 0.01$)$; Wam was significantly reduced in all three treatments (all $P<0.01$ ). There were no significant differences in $\mathrm{Pbm}$ in all groups (Figure 1(a)).

Goblet cell hyperplasia and collagen deposition are implicated in mucus hypersecretion and collagen hyperplasia, which are the major airway remodeling features in asthma and can induce airflow limitation [42, 43]. We observed goblet cell hyperplasia in the airway epithelium of asthmatic rats by the PAS staining, but not in control rats (Figure 1(g)). The number and area of goblet cell were increased significantly in asthma group vs. control group (Figures 1(d) and 1(e); all $P<0.01$ ) and were decreased after drug administration of each group (all $P<0.01$ ), illuminating that the treatment of Bud and EEL could restrain the goblet cell hyperplasia in ovalbumin-induced asthmatic rats either separately or in combination. The inhibitory effect of Bud\&EEL on goblet cell hyperplasia was better than that of Bud or EEL $(P<0.05$ or 0.01$)$.

Collagen deposition in the rat airway wall was observed by Masson's trichrome staining to assess the level of collagenous hyperplasia. The area of collagen fiber was significantly greater in asthma group than that in control group (Figures 1(f) and 1(g); $P<0.01$ ). After treatment, Bud\&EEL notably reduced the area of collagen fiber compared with asthma or Bud group $(P<0.05$ or 0.01$)$. These findings indicate that the combination of Bud and EEL has a better effect on alleviating airway remodeling. 
TABle 2: Antibodies used for WB analysis.

\begin{tabular}{|c|c|c|c|}
\hline Antibody & Description & Concentration & Manufacturer \\
\hline $\mathrm{Bcl}-2$ & Mouse & $1: 700$ & Santa Cruz Biotechnology, Santa Cruz, USA \\
\hline $\mathrm{Bax}$ & Rabbit & $1: 1000$ & Abcam, Cambridge, UK \\
\hline Caspase-3 & Rabbit & $1: 700$ & Abcam, Cambridge, UK \\
\hline Cleaved Caspase-3 & Rabbit & $1: 1000$ & Abcam, Cambridge, UK \\
\hline Beclin-1 & Rabbit & $1: 1000$ & Abcam, Cambridge, UK \\
\hline $\mathrm{LC} 3 \mathrm{~A} / \mathrm{B}$ & Rabbit & $1: 1000$ & Abcam, Cambridge, UK \\
\hline P53 & Mouse & $1: 700$ & Santa Cruz Biotechnology, Santa Cruz, USA \\
\hline mTOR & Rabbit & $1: 2000$ & Abcam, Cambridge, UK \\
\hline p-mTOR & Rabbit & $1: 1000$ & Cell Signaling Technology, Boston, USA \\
\hline
\end{tabular}

TABle 3: Primers used for qPCR analysis.

\begin{tabular}{lrc}
\hline Primer & Forward primer & Reverse primer \\
\hline Bax & CCAAGAAGCTGAGCGAGTGT & TCACGGAGGAAGTCCAGTGT \\
Bcl-2 & AGCATGCGACCTCTGTTTGA & TCACTTGTGGCCCAGGTATG \\
Caspase-3 & TCTACCGCACCCGGTTACTA & CGTACAGTTTCAGCATGGCG \\
P53 & TCGGCTCCGACTATACCACT & GTCCCGTCCCAGAAGATTCC \\
mTOR & GCTTATCAAGCAAGCGACATCTCA \\
Beclin-1 & TTGGCCAATAAGATGGGTCTGAA & TCCACTGGAAGCACAGACCAAG \\
-actin & AGCCATGTACGTAGCCATCC & TGTCAGGGACTCCAGATACGAGTG \\
\hline
\end{tabular}

\subsection{Effects of Bud and EEL on Airway Smooth Muscle Cells} Hyperplasia. High expression of $\alpha$-SMA protein in airway smooth muscle cells is closely associated with airway remodeling and pulmonary fibrosis. Ki-67, regarded as a proliferative marker, plays a key role in epithelial cell proliferation and differentiation [44]. We examined $\alpha$-SMA and Ki-67 by IF to investigate the effect of the coadministration of Bud and EEL on airway smooth muscle cell hyperplasia in ovalbumin-induced asthmatic rat airways (Figure 2(e)).

Positive area and integral optical density (IOD) of $\alpha$-SMA and Ki-67 in asthma group were significantly greater than those in control group (Figures 2(a) and 2(d); all $P<0.01)$. After administration, all three treatments notably inhibited the protein expressions of $\alpha$-SMA and Ki-67 vs. asthma group (all $P<0.01$ ). Bud\&EEL significantly downregulated the two proteins compared with Bud or EEL $(P<0.05$ or 0.01$)$. These data suggest that the effect of combination of Bud and EEL on reducing the airway smooth muscle cells hyperplasia was better than Bud or EEL in asthmatic rats.

3.3. Effects of Bud and EEL on Apoptosis in BALF and Lung Tissue. The high-level apoptosis of epithelial cells and lowlevel apoptosis of immune cells are the important pathogenesis of asthma [12]. In the present study, we used TUNEL assay to detect apoptosis in BALF and lung tissue. Ovalbumin depressed lymphocyte apoptosis in BALF (Figure $3(\mathrm{a}) ; P<0.01$ ), which was determined by IOD of fluorescence labeling. Bud, EEL, and Bud\&EEL significantly induced apoptosis in BALF compared with asthma group (all $P<0.01$ ). In addition, there was a significant difference in apoptosis of BALF between Bud group and Bud\&EEL group $(P<0.01)$.
Similarly, apoptosis activity in lung tissue was inhibited in asthma group, and the inducing apoptosis activity of EEL and Bud\&EEL was found, which was determined by positive area and IOD of fluorescence labeling (Figures 3(b) and 3(c); $P<0.01)$. The effect of Bud\&EEL on inducing apoptosis in lung tissue was better than that of Bud $(P<0.01)$. We can infer from these data that apoptosis in BALF and lung tissue is decreased in ovalbumin-induced asthmatic rats, which can be promoted by the combination of Bud and EEL, suggesting that this positive effect might be associated with improving airway remodeling.

3.4. Effects of Bud and EEL on Bcl-2 and Bax. According to the different biological functions and structural domain, the Bcl2 family, the first discovered cell death regulator, can separate into two types: apoptosis-promoting proteins (Bax, $\mathrm{Bak}$ ) and apoptosis-inhibiting proteins (Bcl-2, Bcl-xL) [45]. In addition, recent studies have indicated that $\mathrm{Bcl}-2$ can not only inhibit autophagy by binding Beclin-1 [46] but also resist glucocorticoid-induced apoptosis [47]. As shown in Figures 4(a) and 4(b), positive area and IOD of Bcl-2 protein detected by IF were increased significantly whereas those of Bax protein were decreased in asthma group vs. control group (all $P<0.01$ ). Compared with asthma group, the positive area of Bcl-2 was remarkably decreased, whereas the positive area and IOD of Bax were increased in all three treatment groups $(P<0.05$ or 0.01$)$; EEL and Bud\&EEL reduced significantly the IOD of $\mathrm{Bcl}-2$ (all $P<0.01$ ). Bud\&EEL notably downregulated Bcl-2 protein and upregulated Bax protein compared with Bud or EEL (all $P<0.01)$.

To further investigate the effects of Bud and EEL on Bcl2 and Bax, we confirmed the protein of Bcl-2 and Bax by WB analysis (Figures 4(c) and 4(e)) and detected the 


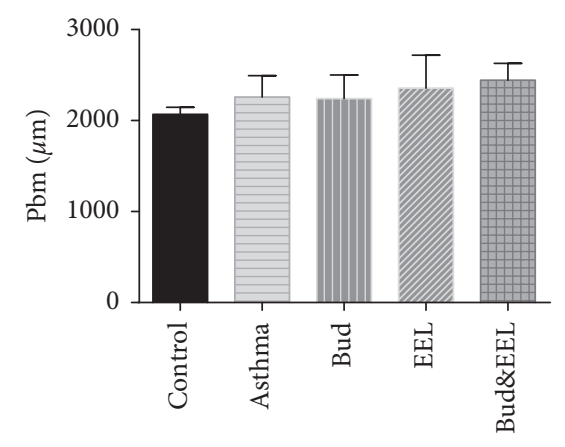

(a)

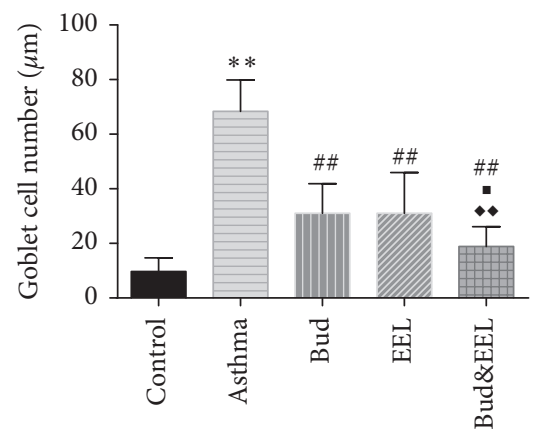

(d)

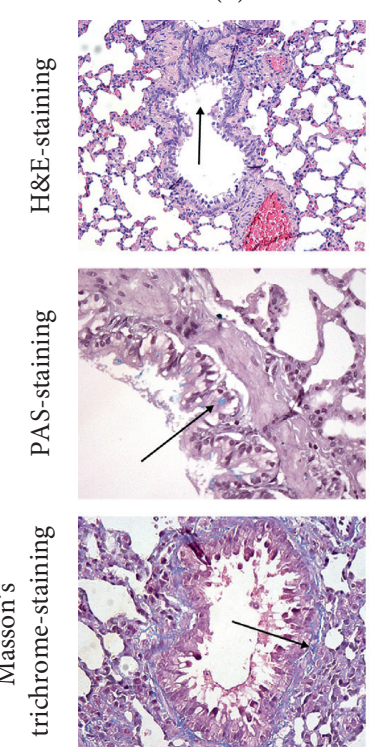

Control

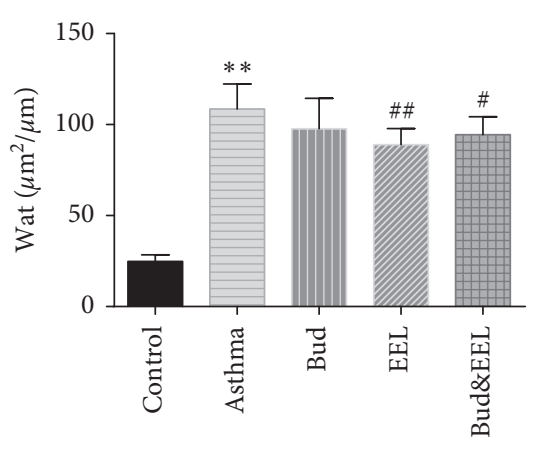

(b)

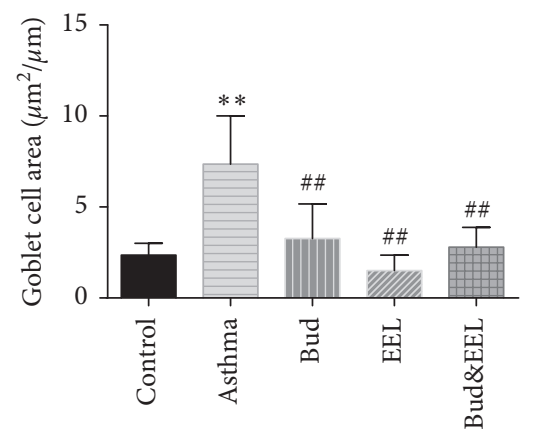

(e)
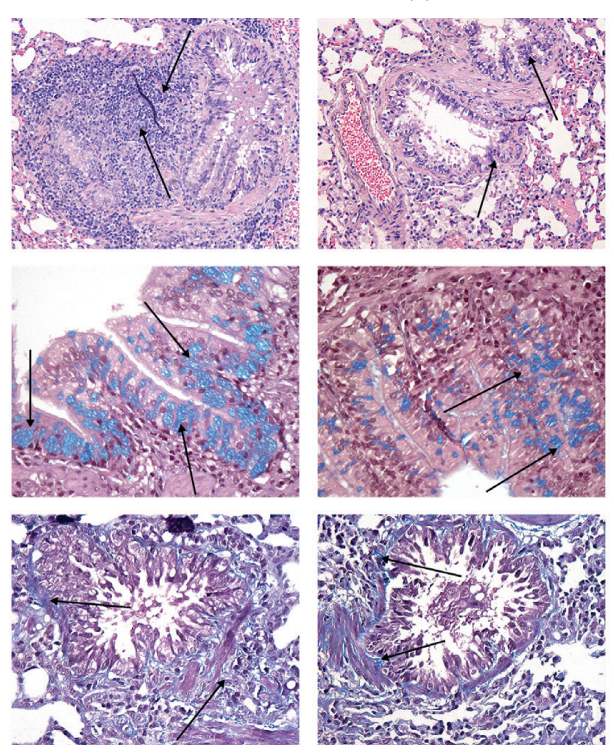

Asthma

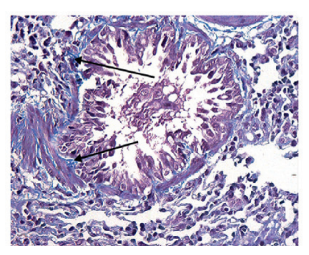

Bud

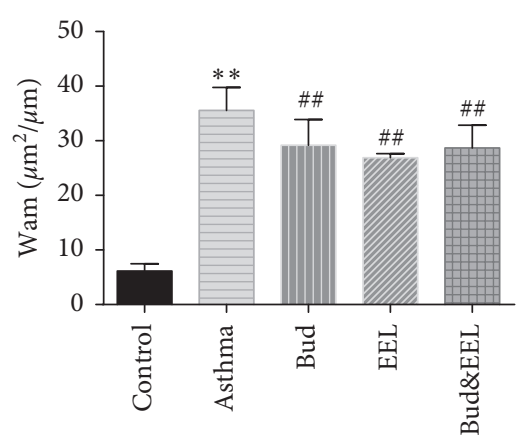

(c)

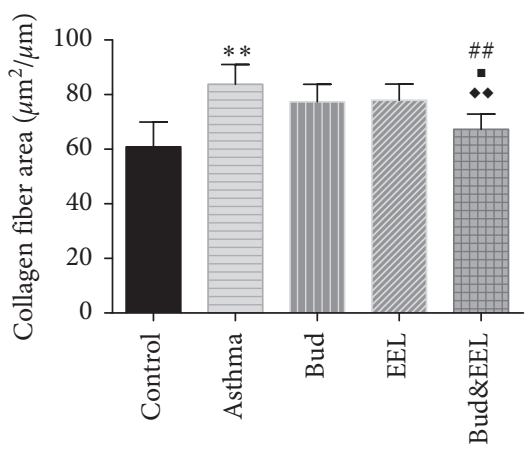

(f)
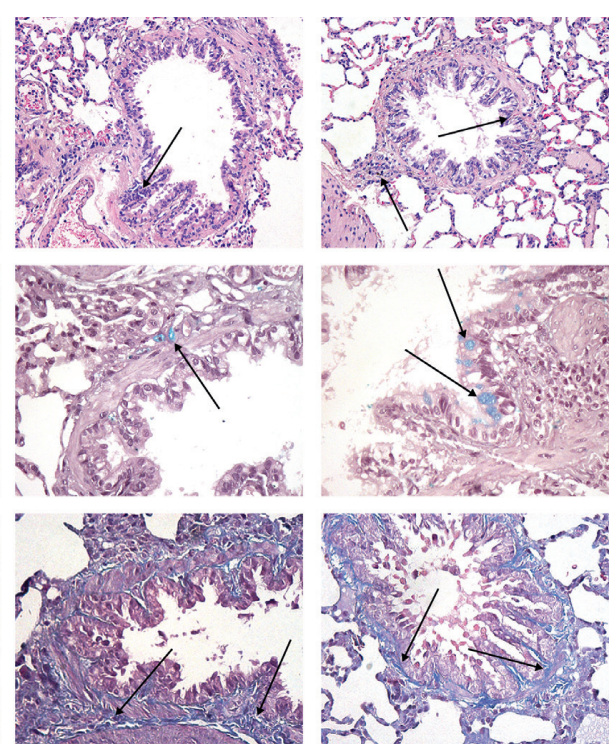

EEL

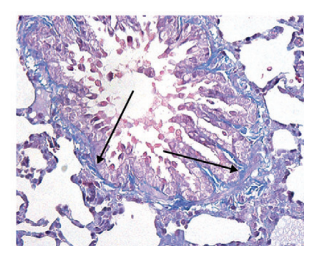

Bud\&EEL

(g)

FIGURE 1: Effects of bud and EEL on airway remodeling. The inflammatory cell infiltration and airway remodeling were observed by (a) the perimeter of basement membrane ( $\mathrm{Pbm}$ ), (b) the standardized thickness of airway wall (Wat), and (c) the standardized thickness of airway smooth muscle (Wam) in H\&E-stained lung sections. The goblet cell hyperplasia was assessed by (d) the number of goblet cell and (e) the area of goblet cell in PAS-stained lung sections. (f) The area of collagen fiber in Masson's trichrome-stained lung sections. (g) Representative photomicrographs of H\&E-stained, PAS-stained, and Masson's trichrome-stained lung sections from each group $(\times 400)$. Data are represented as mean $\pm \mathrm{SD} ; n=6$. $^{* *} P<0.01$ compared with control group; ${ }^{\#} P<0.05$ and ${ }^{\# \#} P<0.01$ compared with asthma group; $P<0.05$ compared with Bud group; $\bullet P<0.01$ compared with EEL group.

mRNA expressions of Bcl-2 and Bax by qPCR (Figure 4(d)). Bcl-2 protein and mRNA were significantly upregulated, while Bax protein and mRNA were downregulated in asthma group vs. control group (all $P<0.01$ ). After administration, all three treatments significantly inhibited the protein and mRNA expression of $\mathrm{Bcl}-2$ and promoted mRNA expression of $\operatorname{Bax}(P<0.05$ or 0.01$)$; EEL and Bud\&EEL upregulated Bax protein (all $P<0.01$ ). We 


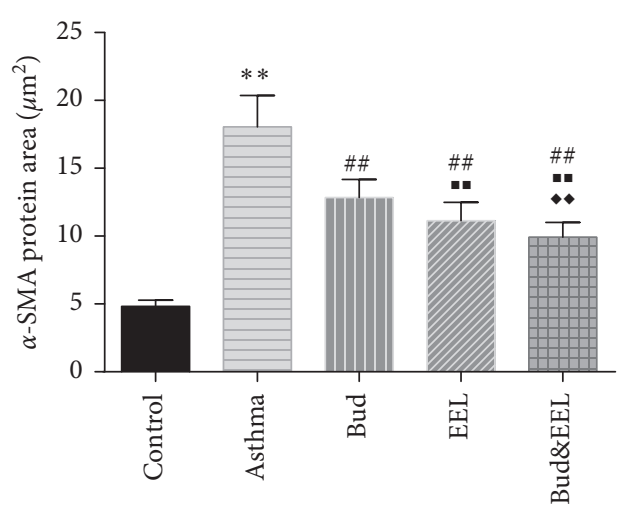

(a)

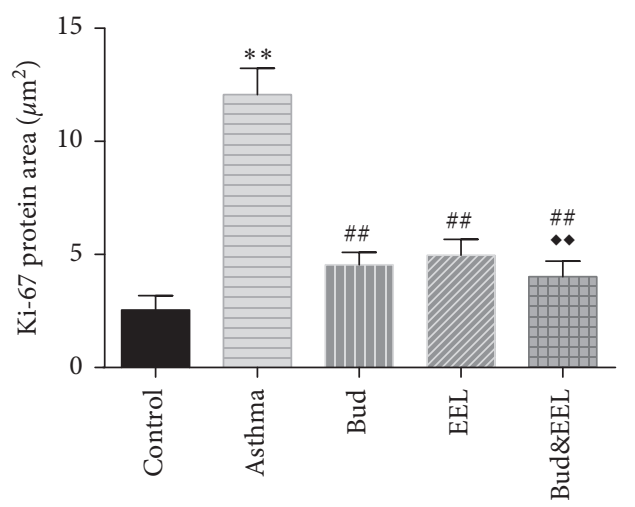

(c)
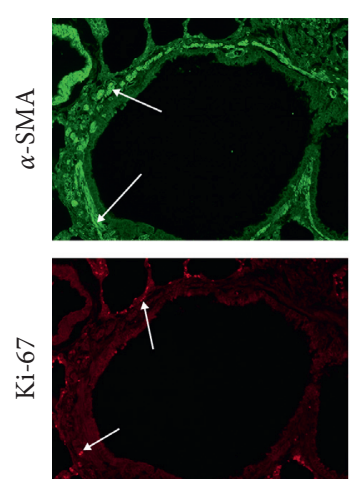

Control
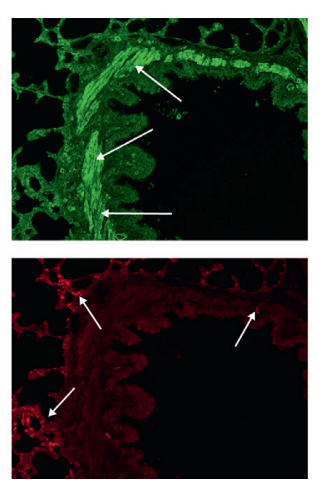

Asthma
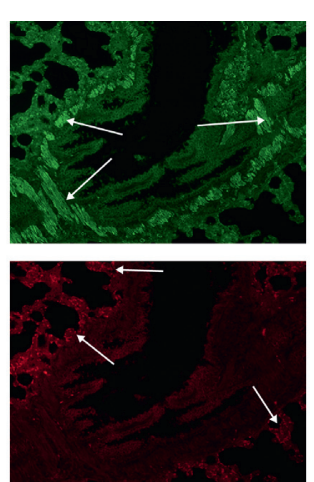

Bud

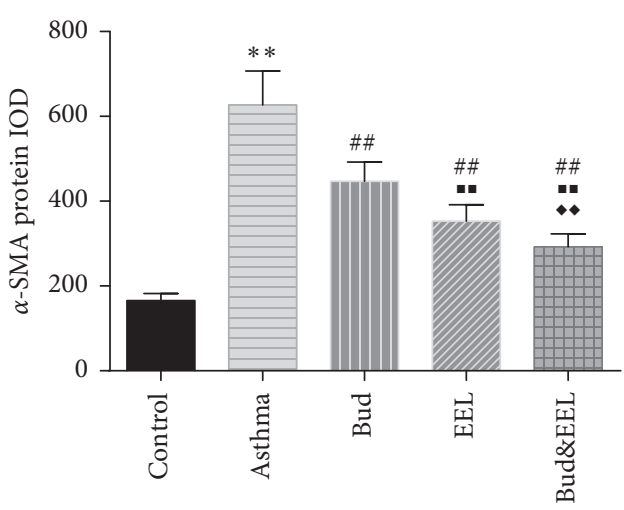

(b)

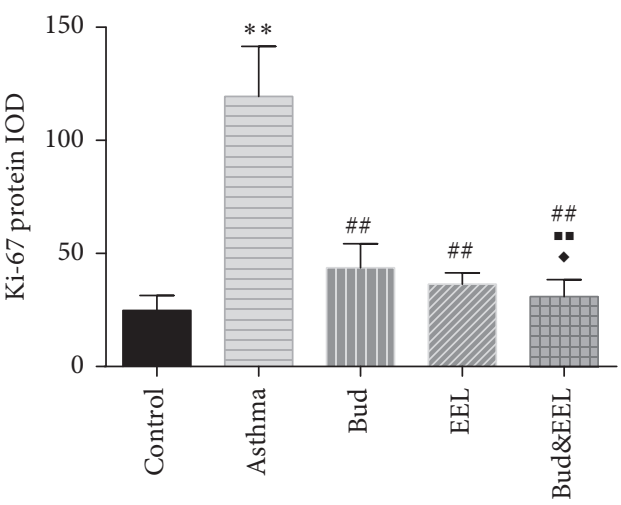

(d)
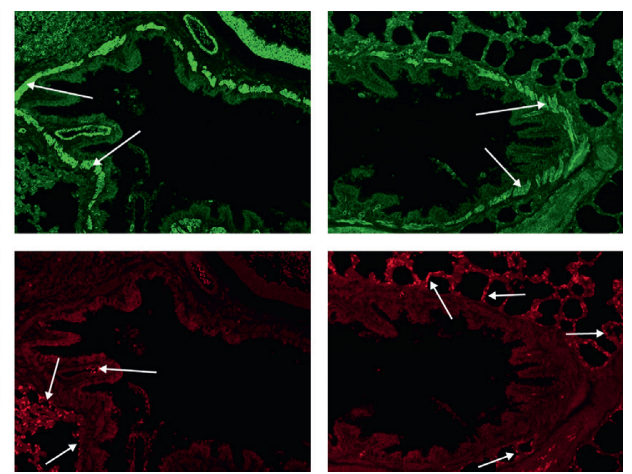

EEL

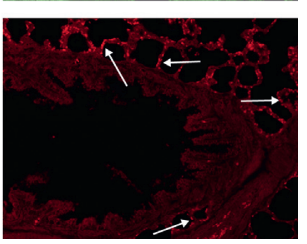

Bud\&EEL

(e)

FIGURE 2: Effects of bud and EEL on cell proliferation in lung tissue. The positive (a) area and (b) IOD of $\alpha$-SMA. The positive (c) area and (d) IOD of Ki-67. (e) Representative immunofluorescence images of $\alpha$-SMA and Ki-67 $(\times 200)$. Data are represented as mean \pm SD; $n=6$. ${ }^{* *} P<0.01$ compared with control group; ${ }^{\# \#} P<0.01$ compared with asthma group; ${ }^{\boldsymbol{*}} P<0.01$ compared with Bud group; $P<0.05$ and $\bullet P<0.01$ compared with EEL group.

observed that Bud\&EEL significantly decreased Bcl-2 protein and increased Bax protein compared with Bud group (all $P<0.01$ ).

The imbalance between the Bcl-2 family proteins, which has been verified in asthmatic rat lung tissues, can impact mitochondria apoptosis pathway [48]. We calculated the protein ratio of $\mathrm{Bcl}-2$ to $\mathrm{Bax}$ measured by $\mathrm{WB}$ to assess the effect of Bud and ELL on balancing the protein expression of Bcl-2 family. The protein ratio of Bcl-2/Bax was notably increased in the asthma group vs. the control group (Figure 4(f); $P<0.05)$. Compared with the asthma or Bud group, Bcl-2/Bax was significantly decreased in EEL and Bud\&EEL groups $(P<0.05$ or 0.01$)$. These results indicate that coadministration of Bud and EEL can regulate $\mathrm{Bcl}-2$ and $\mathrm{Bax}$ and balance Bcl-2/Bax in asthmatic rats. Further, EEL has synergistic effect on regulating Bcl-2 and Bax with Bud.

3.5. Effects of Bud and EEL on Caspase-3 and Cleaved Caspase3. Caspase-3 is an executor caspase, playing an essential role in the final phase of apoptosis [49]. Cleaving proCaspase- 3 to 


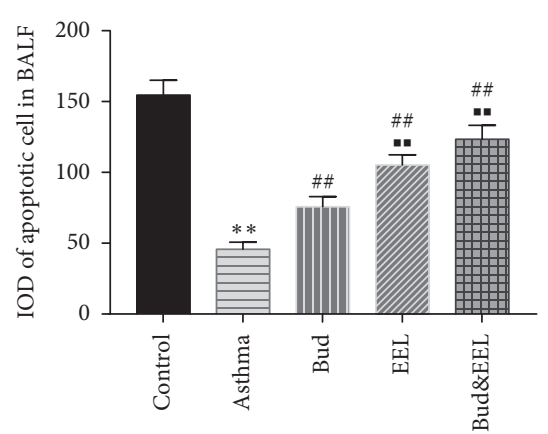

(a)

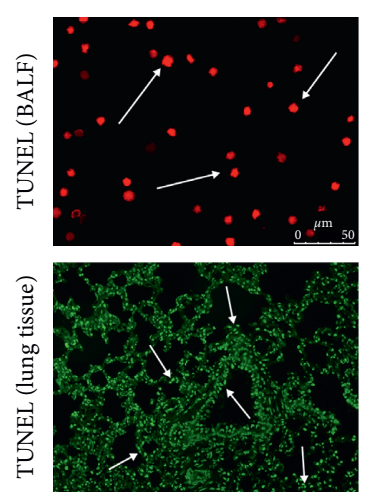

Control

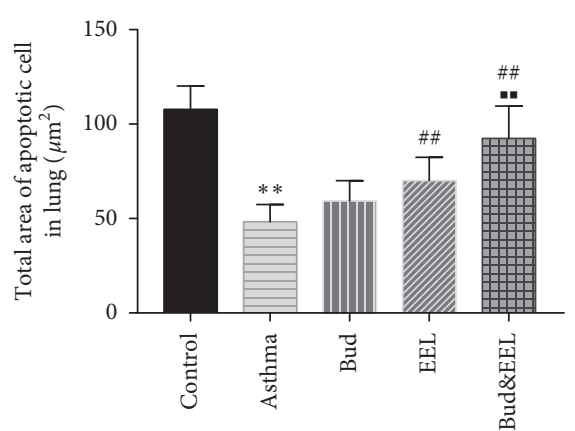

(b)
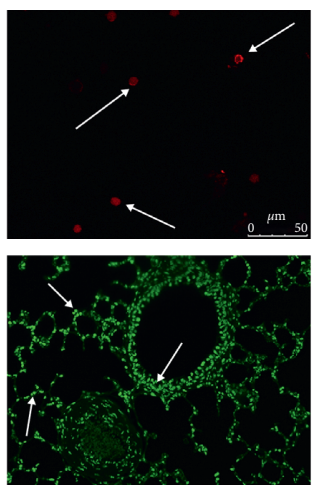

Asthma
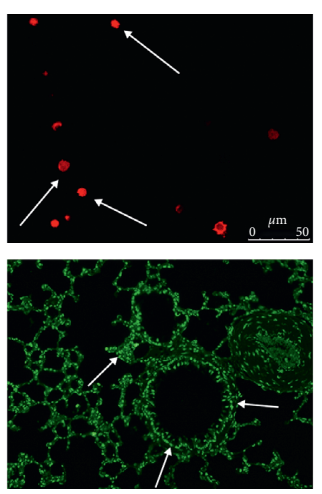

Bud

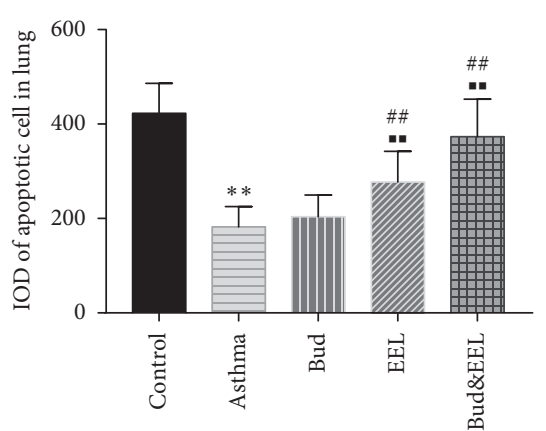

(c)
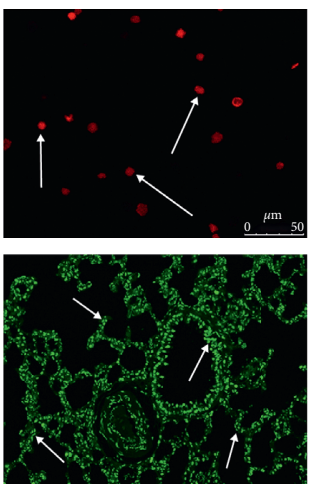

EEL

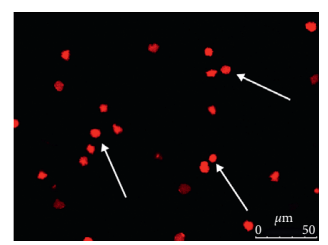

(d)

Figure 3: Effects of bud and EEL on apoptosis in BALF and lung tissue. (a) The IOD of fluorescein-dUTP in BALF cells. The positive (b) area and (c) IOD of fluorescein-dUTP in lung tissue. (d) Representative immunofluorescence images of TUNEL in BALF $(\times 400)$ and in lung tissue $(\times 200)$. Red: apoptosis in BALF; green: apoptosis in lung tissue. Data are represented as mean $\pm \mathrm{SD} ; n=6 .{ }^{* *} P<0.01$ compared with control group; ${ }^{\#} P<0.01$ compared with asthma group; $\quad P<0.01$ compared with Bud group.

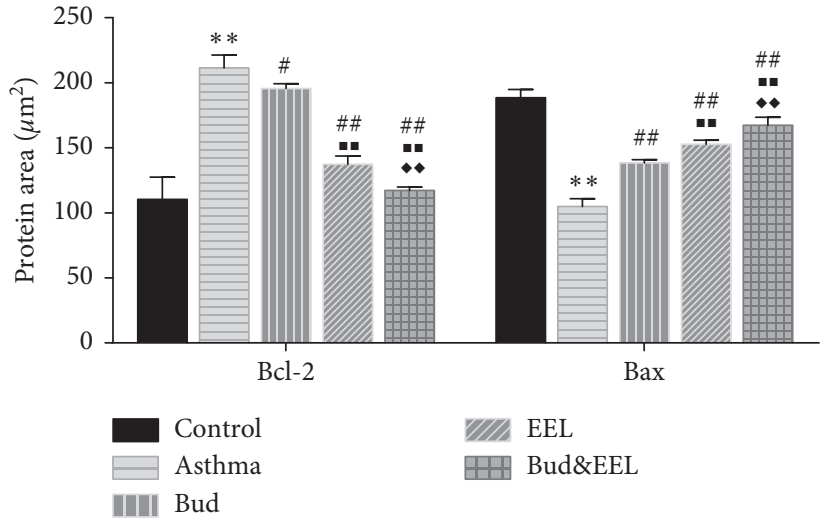

(a)

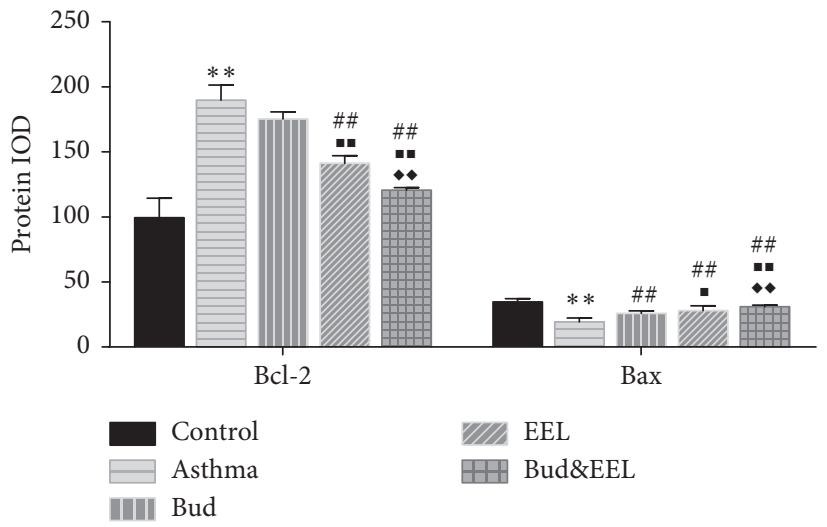

(b)

Figure 4: Continued. 


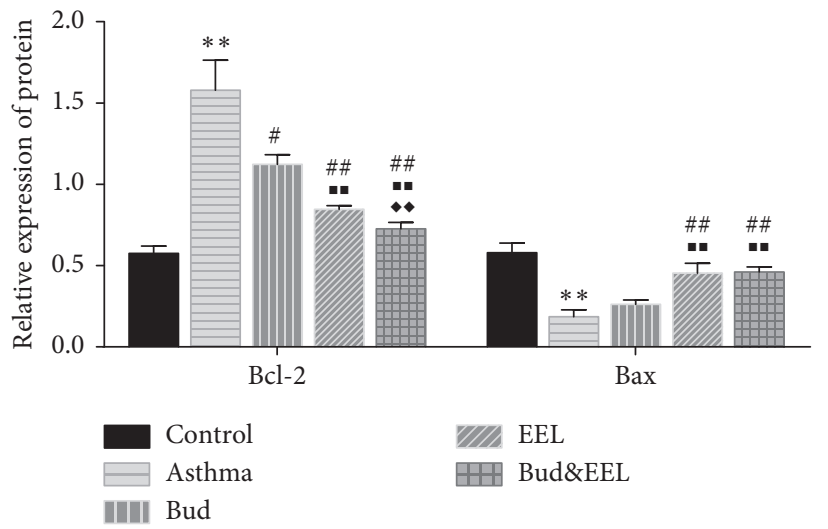

(c)

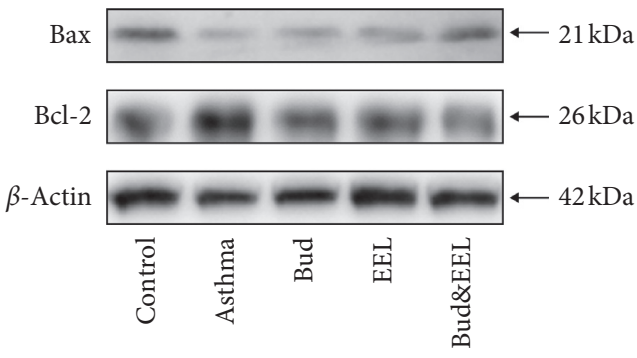

(e)
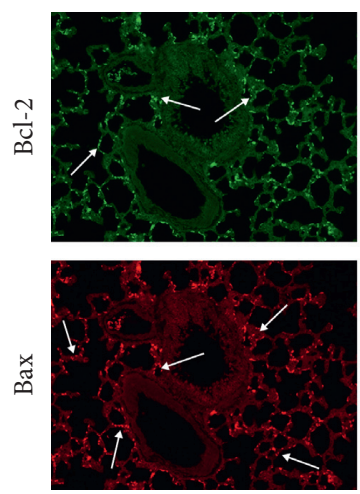

Control
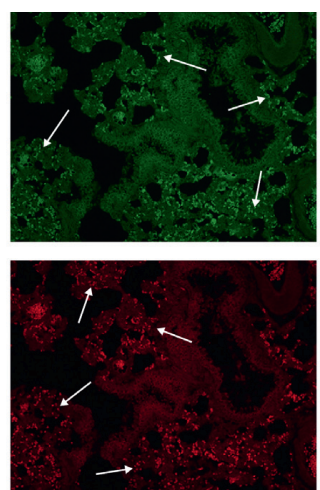

Asthma

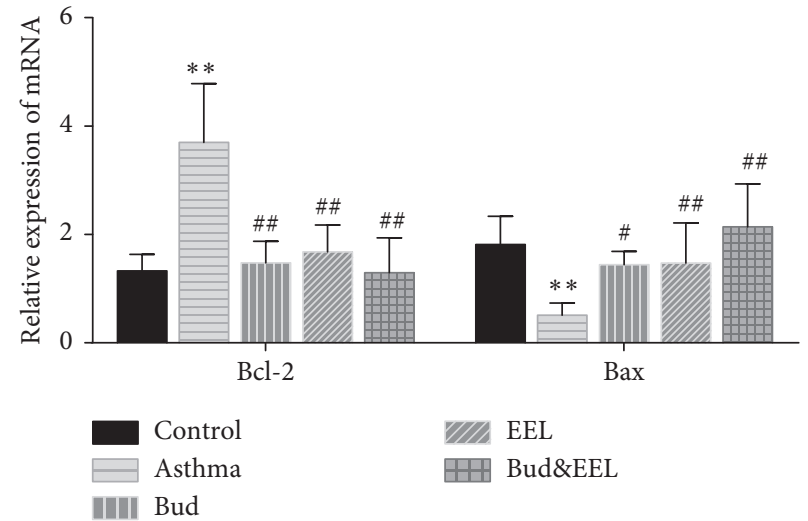

(d)

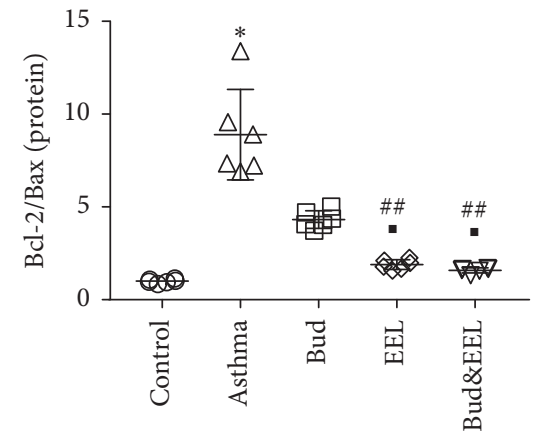

(f)
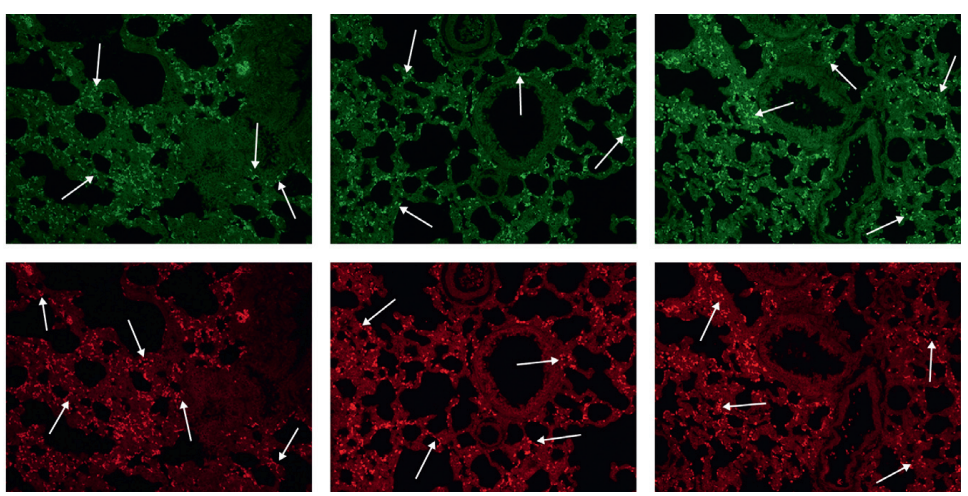

Bud

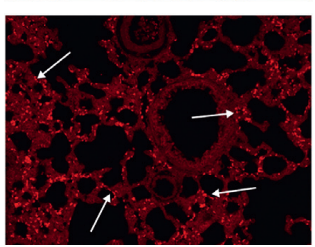

EEL

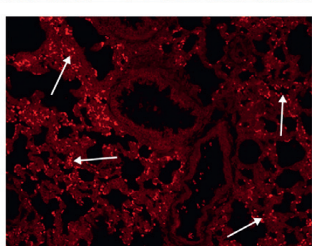

Bud\&EEL

(g)

FIgure 4: Effects of bud and EEL on Bcl-2 and Bax. The protein area (a) and IOD (b) of Bcl-2 and Bax were measured by immunofluorescence staining. (c) Summarized data of mean density of Bcl-2 and Bax protein expression in lung tissues measured by WB, normalized to $\beta$-actin. (d) The mRNA expressions of Bcl-2 and Bax were measured by qPCR analysis with $\beta$-actin as an internal control. (e) Representative WB photographs of Bcl-2 and Bax were viewed, and $\beta$-actin was used for normalization. (f) The ratio of Bcl-2 to Bax was calculated in the level of relative protein measured by WB. (g) Representative immunofluorescence images of Bcl-2 and Bax ( $\times 200)$. Data are represented as mean \pm SD; $n=6 .{ }^{*} P<0.05$ and ${ }^{* *} P<0.01$ compared with control group; ${ }^{\#} P<0.05$ and ${ }^{\# \#} P<0.01$ compared with asthma group; $P<0.05$ and $\boldsymbol{\top} P<0.01$ compared with bud group; ${ }^{\star} P<0.01$ compared with EEL group.

generate the active Caspase- 3 heterodimer (cleaved Caspase- 3) is a necessary step in activating apoptosis [50]. In addition, Caspase-3, as a regulator of autophagy, can inhibit autophagy through cleaving autophagy-related gene (ATG), such as ATG3 and ATG4 [51, 52].

We observed that Caspase- 3 protein expression detected by IF was significantly reduced in the asthma group vs. the control group (Figures 5(a) and 5(b); all $P<0.01$ ) and was increased after drug administration of each group (all $P<0.01$ ); Caspase- 3 mRNA expression by qPCR was significantly decreased in asthma group vs. control group (Figure 5(c); $P<0.01$ ) and was upregulated after the treatment of EEL and Bud\&EEL $(P<0.05$ or 0.01$)$.

To further investigate the effects of Bud and EEL on Caspase- 3 protein, we used WB analysis to detect Caspase- 3 and cleaved Caspase-3 (Figures 5(d) and 5(e)). We found 


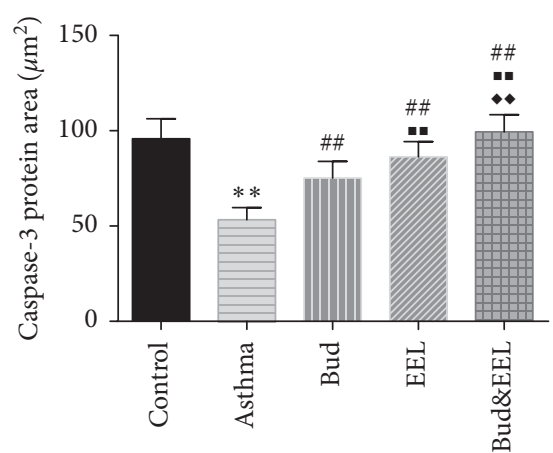

(a)

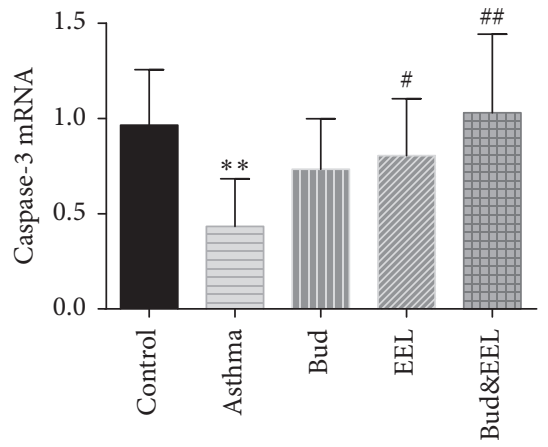

(c)

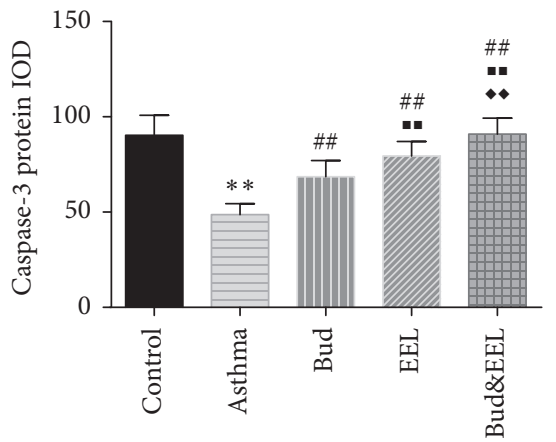

(b)

Cleaved caspase- 3

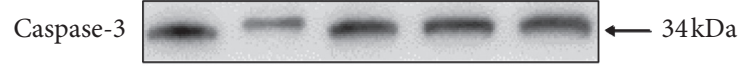

$\beta$-Actin

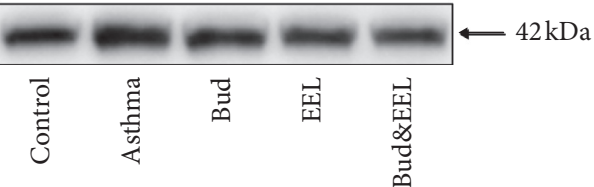

(d)

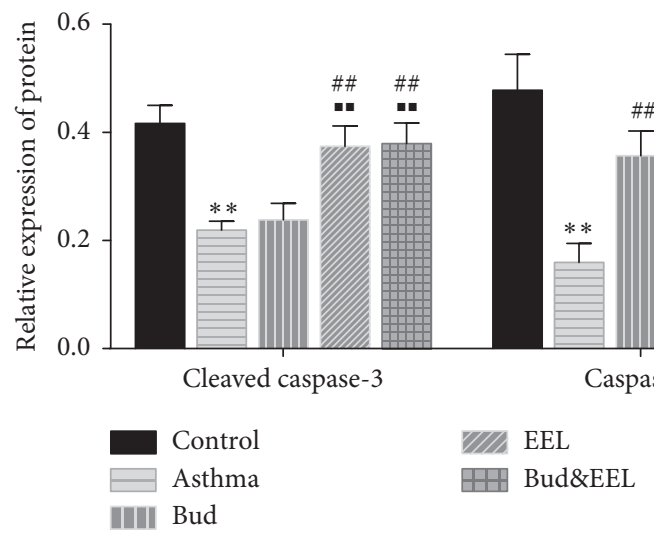

(e)

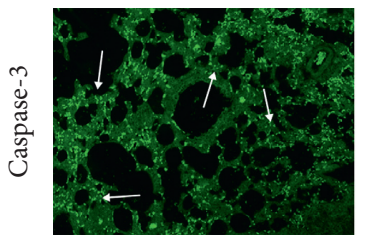

Control

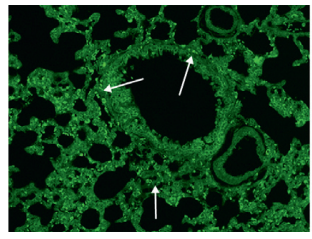

Asthma

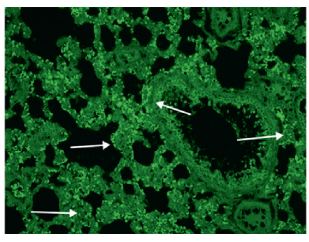

Bud

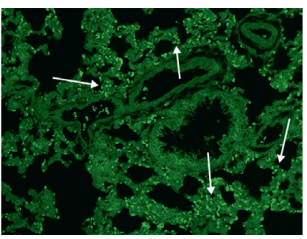

EEL

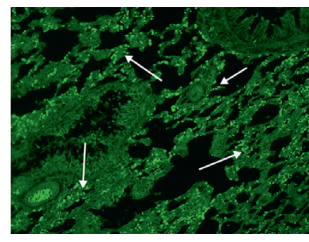

Bud\&EEL

(f)

Figure 5: Effects of bud and EEL on Caspase-3 and cleaved Caspase-3. The protein area (a) and IOD (b) of Caspase-3 were measured by immunofluorescence staining. (c) The mRNA expressions of Caspase- 3 were measured by qPCR analysis with $\beta$-actin as an internal control. (d) Representative WB photographs of Caspase-3 and cleaved Caspase-3 were viewed, and $\beta$-actin was used for normalization. (e) Summarized data of mean density of cleaved Caspase- 3 and Caspase- 3 protein expression in lung tissues measured by WB, normalized to $\beta$-actin. (f) Representative immunofluorescence images of Caspase-3 $(\times 200)$. Data are represented as mean \pm SD; $n=6 .{ }^{* *} P<0.01$ compared with control group; ${ }^{\#} P<0.05$ and ${ }^{\# \#} P<0.01$ compared with asthma group; $\boldsymbol{\|} P<0.01$ compared with Bud group; ${ }^{\bullet} P<0.01$ compared with EEL group. 
that Caspase-3 and cleaved Caspase- 3 were reduced significantly in the asthma group vs. the control group (all $P<0.01)$. EEL and Bud\&EEL significantly promoted cleaved Caspase-3 protein, and all three treatments remarkably promoted Caspase- 3 protein compared with asthma group (all $P<0.01$ ). These results suggest that the coadministration of Bud and EEL has a better effect on activating Caspase-3 than single-use Bud treatment.

3.6. Effects of Bud and EEL on LC3 and Beclin-1. The macroautophagy, a primary form of autophagy, participates in asthmatic pathogenesis. LC3 and Beclin-1 proteins can promote autophagy by taking part in the formation of autophagosome which is an important procedure of macroautophagy $[53,54]$. To investigate the effects of Bud and EEL on autophagy, we detected Beclin-1 and LC3.

Beclin-1 protein detected by both IF and $\mathrm{WB}$, and Beclin-1 mRNA detected by qPCR were increased significantly in asthma group vs. control group (Figures 6(a), 6(d), and 6(f); all $P<0.01$ ), and were decreased after drug administration of each group (all $P<0.01$ ). Compared with Bud group, Beclin-1 protein was downregulated remarkably in EEL and Bud\&EEL groups (all $P<0.01$ ).

We measured the protein expressions of LC3 by WB and calculated the ratio of LC3-II to LC3-I. LC3-II/LC3-I was upregulated remarkably in the asthma group vs. the control group (Figures 6(d) and 6(e); $P<0.01$ ). After administration, all three treatments notably reduced LC3-II/LC3-I compared with asthma group (all $P<0.01$ ), indicating that the activity of autophagy in asthmatic rat lung tissue was promoted, which could be resisted with the treatment of Bud and EEL. In addition, there was significant decrease in EEL and Bud\&EEL groups compared with Bud group (all $P<0.01)$. These results suggested that the inhibitory effect of Bud\&EEL on autophagy was better than that of single-Bud treatment in ovalbumin-induced rats, indicating that might be a potential mechanism of synergistic effect of EEL with Bud on airway remodeling.

3.7. Effects of Bud and EEL on P53, mTOR, and p-mTOR. The relationship between apoptosis and autophagy is very complicated, because some proteins can influence the activity of both apoptosis and autophagy such as P53 and mTOR. P53 can promote the expression of many proapoptotic proteins such as Bax, Apaf-1, and Caspase- 6 and ultimately induce apoptosis. What is more, nuclear p53 promotes autophagy, whereas cytoplasmic p53 prevents it. Both functions work through impacting AMPK-TSC2mTOR pathway. mTOR inhibits autophagy and apoptosis and affects cell cycle regulation, proliferation, and differentiation $[55,56]$. mTORC1 and mTORC2 are two complexes formed by mTOR. Phospho-mTOR (Ser2448) is mainly regulated by $\mathrm{mTORC} 1$ which can downregulate autophagy.

We found that the protein and mRNA of P53 and mTOR, detected by IF and qPCR, were significantly reduced in the asthma group vs. the control group (Figures 7(a), 7(b), and $7(\mathrm{e})$; all $P<0.01)$. Compared with asthma group, P53 and $\mathrm{mTOR}$ in both protein and mRNA level were upregulated significantly in all three treatment groups $(P<0.05$ or 0.01$)$. The effect of Bud\&EEL on upregulating P53 and mTOR was better than that of Bud $(P<0.05$ or 0.01$)$.

To further confirm the effects of the Bud and EEL on P53 and $\mathrm{mTOR}$, we detected the protein expressions of P53, mTOR, and $\mathrm{p}-\mathrm{mTOR}$ in rat lung tissue by WB analysis (Figures $7(\mathrm{c})$ and $7(\mathrm{~d})$ ). The protein expressions of P53, mTOR, and p-mTOR were reduced remarkably in asthma group vs. control group (all $P<0.01$ ) and were increased after drug administration of each group (all $P<0.01$ ). Bud\&EEL significantly increased the protein expression of P53, mTOR, and p-mTOR compared with Bud $(P<0.05$ or 0.01$)$. These results suggest that EEL could activate P53 and mTOR in asthmatic rat lung tissue, which might be associated with the synergistic effect of EEL with Bud on airway remodeling.

\section{Discussion}

Airway remodeling is an irreversible change in airway structure and function induced by chronic impairment, relating to severity of asthma $[57,58]$. At present, asthmatic treatments (including GCs and long-acting $\beta 2$-agonist and bronchodilators) have little influence on airway remodeling, the primary cause of refractory asthma [59]. In our study, Bud\&EEL relieved the incrassation of the airway wall and smooth muscle, goblet cell hyperplasia, and collagen deposition in ovalbumin-induced asthma rats. In addition, Bud\&EEL significantly reduced goblet cell number and alleviated collagen deposition, compared with Bud. These findings indicate that the combination of EEL with Bud might have a better effect on the pathological changes of airway remodeling, especially the collagen deposition and goblet cell hyperplasia.

Airway smooth muscle cells are not only the secondary effector cells but also the incipient inductor cells in airway remodeling [4]. $\alpha$-SMA reflects the scale and shrink capability of smooth muscle cells which induce tracheal stenosis and contraction [60], highly expressed in myofibroblasts [61] and negatively correlated with lung function in vitro asthmatic experiments [62]. Ki-67 is regarded as a crucial marker of cell proliferation which is highly expressed in cycling cells but lowly expressed in resting Go cells $[63,64]$. In order to further confirm the effect of Bud\&EEL on airway remodeling, we investigated hyperplasia of airway smooth muscle cells by detecting $\alpha$-SMA and Ki-67 in lung tissue. We found that $\alpha$-SMA and Ki- 67 were remarkably increased in asthma rats, consistent with the pathological changes of airway remodeling. EEL, Bud, and Bud\&EEL significantly reduced $\alpha$-SMA and $\mathrm{Ki}-67$. Bud\&EEL had the priority in downregulating $\alpha$-SMA and Ki-67 compared to Bud, suggesting that the coadministration of EEL and Bud has synergistic effects on airway smooth muscle cell hyperplasia in ovalbumin-induced asthma rats.

As an apoptosis inducer, GCs' antiremodeling effect is related to regulating apoptosis. We found that Bud\&EEL reduced apoptosis in BALF and lung tissue, and this 


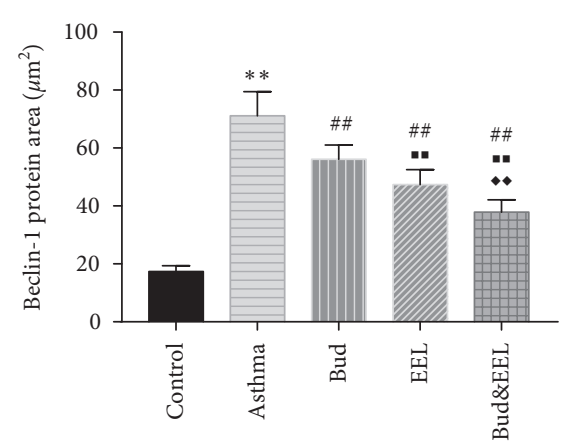

(a)

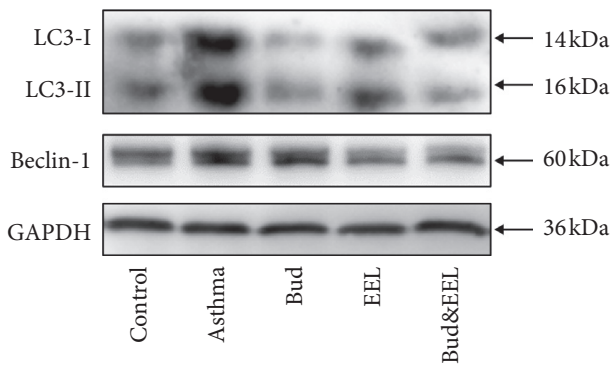

(d)

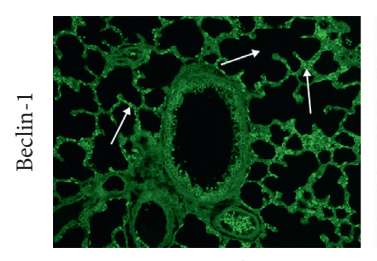

Control

Asthma

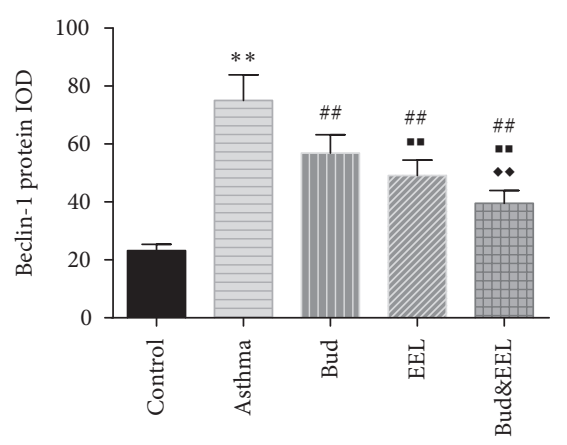

(b)

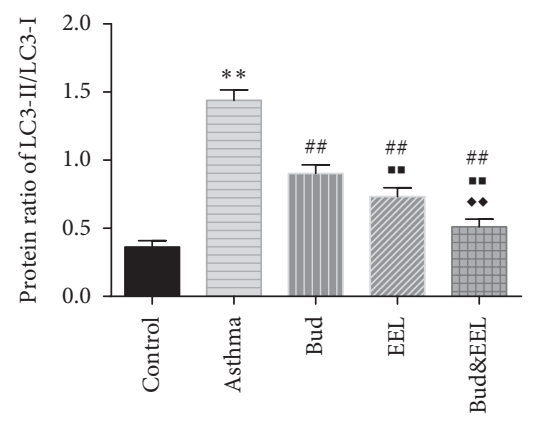

(e)

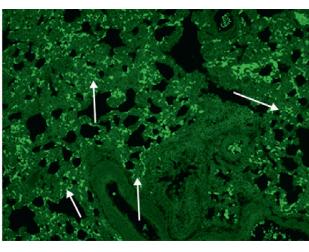

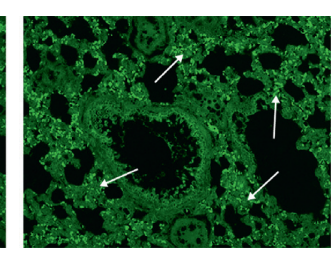

Bud

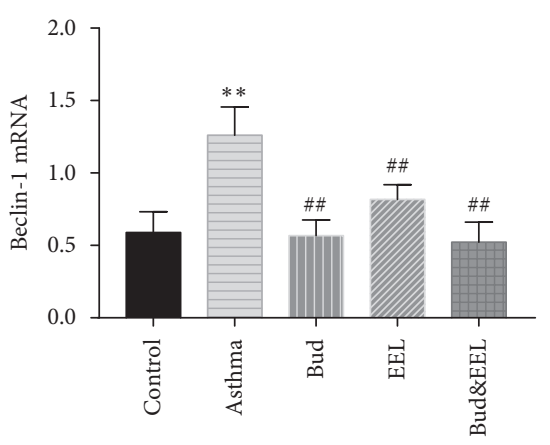

(c)

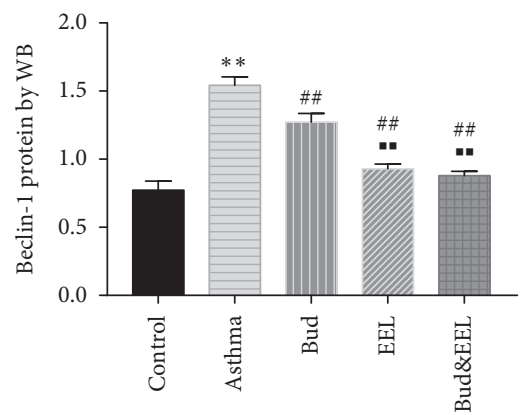

(f)

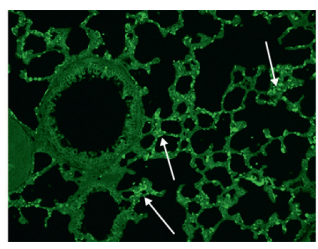

EEL

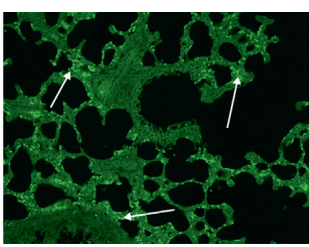

Bud\&EEL

(g)

FIGURE 6: Effects of Bud and EEL on LC3 and Beclin-1. The protein area (a) and IOD (b) of Beclin-1 were measured by immunofluorescence staining. (c) The mRNA expressions of Beclin-1 were measured by qPCR analysis with $\beta$-actin as an internal control. (d) Representative WB photographs of LC3 and Beclin-1 were viewed, and GAPDH was used for normalization. Summarized data of mean density of protein ratio of LC3-II/LC3-I (e) and Beclin-1 protein (f) in lung tissues measured by WB, normalized to GAPDH. (g) Representative immunofluorescence images of Beclin-1 $(\times 200)$. Data are represented as mean \pm SD; $n=6{ }^{* *} P<0.01$ compared with control group; \#\# $P<0.01$ compared with asthma group; $\boldsymbol{M} P<0.01$ compared with Bud group; ${ }^{\bullet} P<0.01$ compared with EEL group.

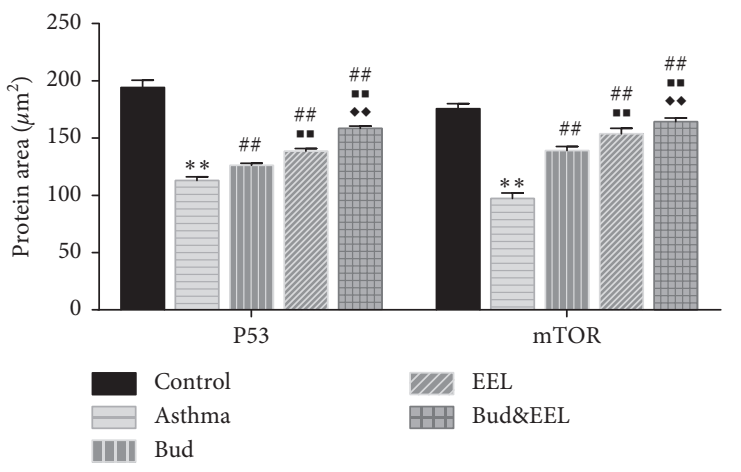

(a)

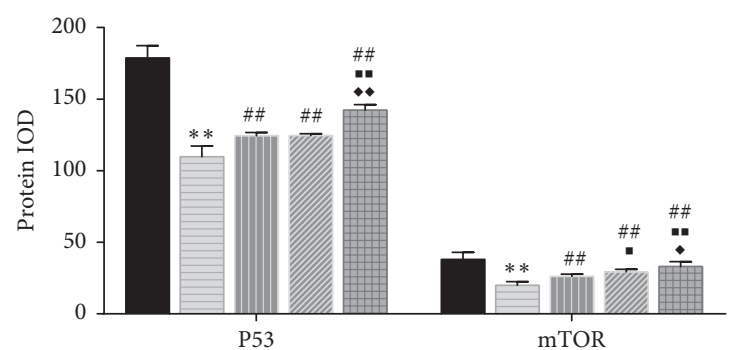

VIIS EEL

BHAEEL

(b)

Figure 7: Continued. 


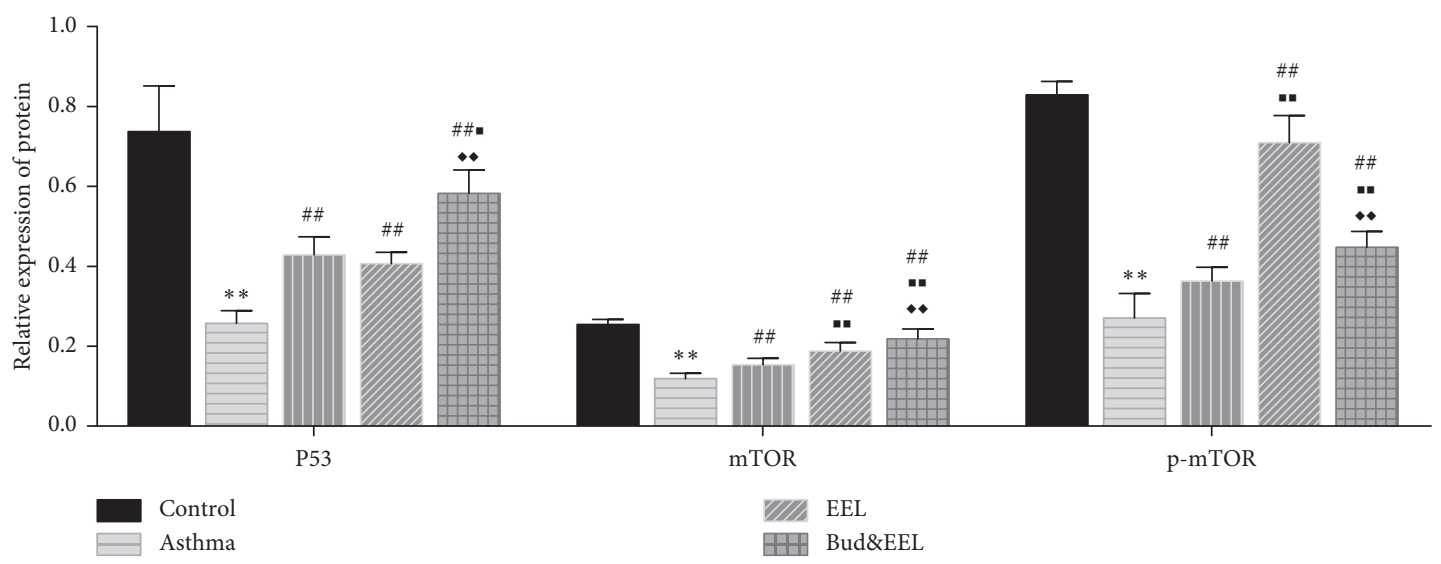

(c)
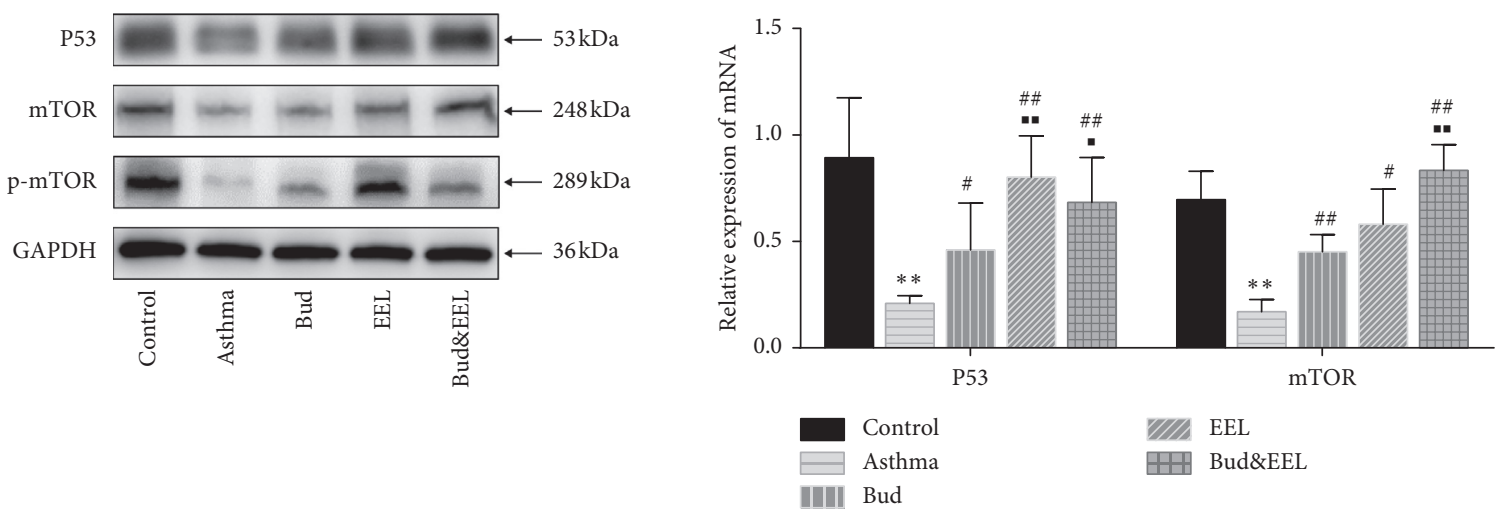

(d)
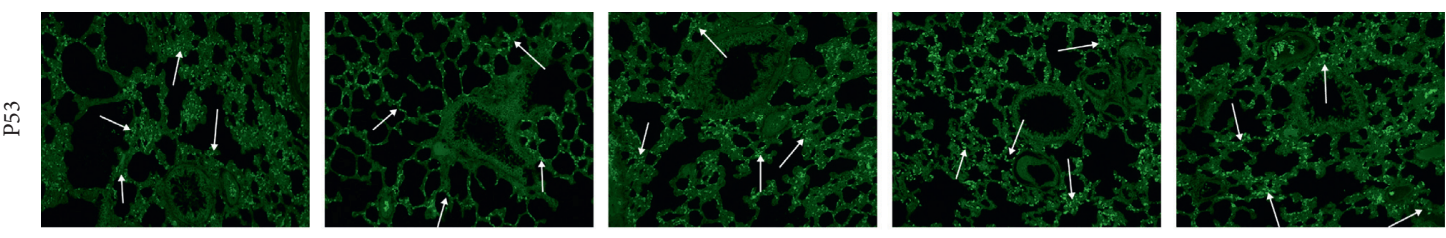

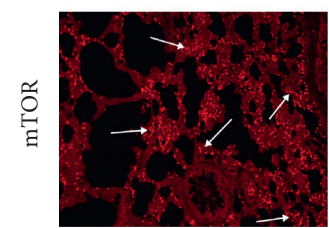

Control

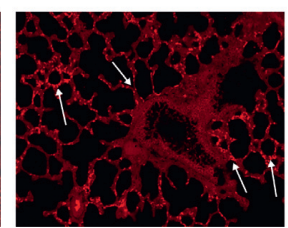

Asthma

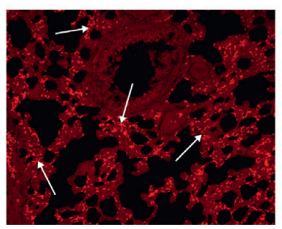

Bud

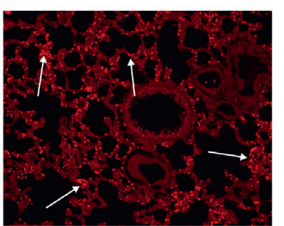

EEL

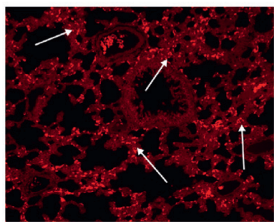

Bud\&EEL

(f)

FIgURe 7: Effects of Bud and EEL on P53, mTOR, and p-mTOR. The protein area (a) and IOD (b) of P53 and mTOR were measured by immunofluorescence staining. (c) Summarized data of mean density of P53, mTOR, and p-mTOR protein expression in lung tissues measured by WB, normalized to GAPDH. (d) Representative WB photographs of P53, mTOR, and p-mTOR were viewed, and GAPDH was used for normalization. (e) The mRNA expressions of P53 and mTOR were measured by qPCR analysis with $\beta$-actin as an internal control. (f) Representative immunofluorescence images of P53 and mTOR $(\times 200)$. Data are represented as mean \pm SD; $n=6$. ${ }^{* *} P<0.01$ compared with control group; ${ }^{\#} P<0.05$ and ${ }^{\# \#} P<0.01$ compared with asthma group; $P<0.05$ and $P<0.01$ compared with Bud group; $P<0.05$ and $P<0.01$ compared with EEL group.

influence was more effective than that of single-Bud treatment. In order to investigate the molecular mechanism of that, we detected Bcl-2, Bax, Caspase-3, and P53 in protein and mRNA. In the Bcl-2 protein family, Bcl-2 (apoptosisinhibiting protein) and Bax (apoptosis-promoting protein) play important roles in mitochondrial apoptosis pathway by regulating the mitochondrial release of cytochrome $c$ $[65,66]$. In the Caspases family, Caspase- 3 is a key protease in mitochondria-dependent and -independent apoptosis pathways, executing final phase of apoptosis [67, 68]. Remarkably, Bcl-2 and Bax are upstream regulators of Caspase3 in intrinsic apoptosis pathway $[69,70]$. P53, playing an 


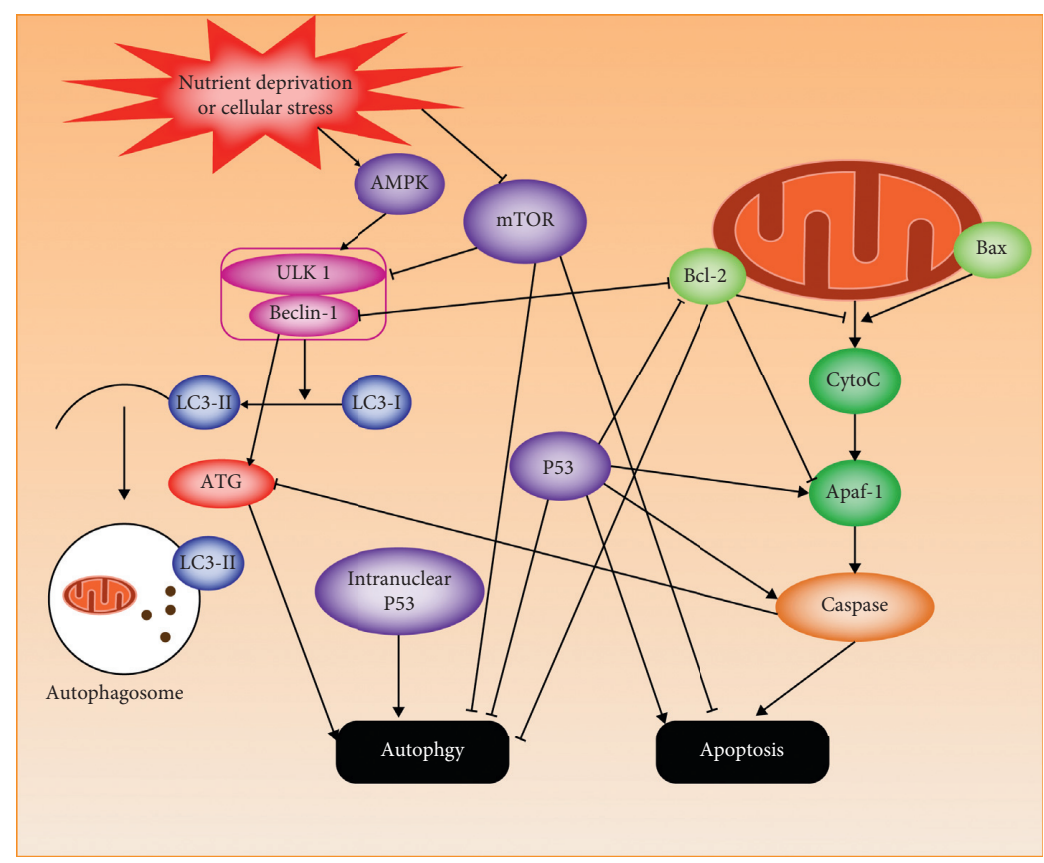

(a)

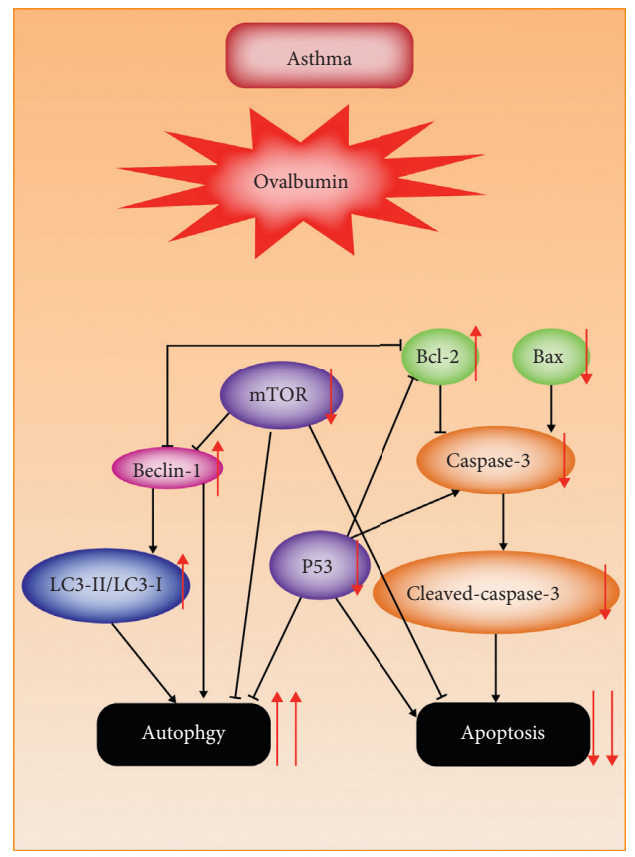

(b)

FIGURE 8: The crosstalk between autophagy and apoptosis. (a) In physiological situations, the activities of autophagy and apoptosis are influenced by many molecular signaling pathways, and some protein can regulate both autophagy and apoptosis. (b) In ovalbumin-induced asthmatic rats, the activity of autophagy is increased whereas apoptosis is reduced through detecting the expressions of autophagy-related proteins and apoptosis-related proteins.

essential role in mediating apoptosis, cell cycle arrest, and DNA repair, can improve the activity of apoptosis by upregulating proapoptosis genes [71]. For example, P53 can bind to Bcl-2, promoting the release of Bax and enhancing the activity of Caspase-3 [72, 73]. We observed that ovalbumin depressed apoptosis activity and proapoptosis factors including Bax, Caspase-3, and P53 increased Bcl-2 and Bcl2/Bax. Bud, EEL, and Bud\&EEL upregulated apoptosis in BALF, Bax, and P53 in protein and mRNA levels but reduced $\mathrm{Bcl}-2$. In addition, EEL and Bud\&EEL could upregulate apoptosis in lung tissue and the expressions of cleaved Caspase- 3 and Caspase- 3 but decrease Bcl-2/Bax. Moreover, Bud\&EEL had better effects on regulating apoptosis, Bcl-2, Bax, P53, and Caspase-3 than Bud. The data suggest that promoting apoptosis might be a possible mechanism by which the coadministration of Bud and EEL improves airway remodeling in asthma.

Autophagy plays a pivotal role in maintaining cellular homeostasis through elimination of misfolded proteins, protein aggregates, and damaged organelles in physiology situations [74]. It can be divided into three categories: microautophagy, chaperone-mediated autophagy (CMA), and macroautophagy. Macroautophagy (hereafter referred to as "autophagy") is implicated in airway inflammation and airway remodeling in asthma [20]. In mediating airway remodeling process, autophagy in airway epithelial cells can induce epithelial-mesenchymal transition in airways, leading to aggravation of airway remodeling and pulmonary fibrosis [75]. In order to observe the autophagic activity in asthmatic rat and the effects of Bud and EEL on that, we detected the expressions of Beclin-1, LC3, and mTOR which play crucial roles in regulating autophagy. Under conditions of nutrient deprivation or cellular stress, AMPK activates phosphorylation of ULK and Beclin-1, triggering autophagy [76]; mTORC1 is a complex of mTOR which can catalyze the inactivating phosphorylation of ULK1, suppressing autophagy [77]. Beclin-1 can activate ATG and promote the conversion of cytoplasmic soluble LC3-I to membrane combined LC3-II to accelerate formation of autophagosome, inducing autophagy. Therefore, the protein ratio of LC3-II to LC3-I is regarded as the autophagic activity [78]. We found that Beclin-1 and LC3-II/LC3-I were increased while mTOR and $\mathrm{p}$-mTOR were decreased in ovalbumin-induced asthma model rats. EEL, Bud, and Bud\&EEL could reduce Beclin-1 and LC3-II/LC3-I but promote mTOR and p-mTOR. In addition, the effect of Bud\&EEL on regulating autophagy was better than that of single-use Bud. Our study indicates that Bud\&EEL has synergistic effect on inhibiting autophagy induced in asthma rats, suggesting that inhibiting autophagy might be a possible mechanism by which the coadministration of Bud and EEL improves airway remodeling.

As shown in Figure 8, autophagy has many crosstalks with apoptosis due to the interactions of autophagy- and apoptosis-related proteins [79], including the following: (i) the interaction between Bcl-2 and Beclin-1 inhibits autophagy. (ii) P53 exerts different effects on autophagy, according to the location of P53 in cell-nuclear p53 promotes it, whereas cytoplasmic p53 prevents it. $\mathrm{P} 53$ promotes apoptosis by increasing the expression of proapoptotic proteins such as Bax, Apaf-1, and Caspase- 6 which mediates 
intrinsic and extrinsic apoptosis pathway. (iii) mTOR acts as an inhibitor on both autophagy and apoptosis. (iv) Activated caspases can inhibit autophagy by splitting autophagy-associated proteins, such as Beclin-1, ATG5, and p62 [80, 81]. In the present study, we observed that the mRNA and protein expressions of Bcl-2, Bax, Caspase-3, P53, Beclin-1, and mTOR make crosstalk between apoptosis and autophagy clear in asthma rats. Our study shows that apoptotic activity is decreased whereas autophagic activity is increased, and the interaction between them exists in ovalbumin-induced asthma rats. It confirms that disordered apoptosis and autophagy are asthmatic pathogenesis, providing researchers with ideas on the development of new drugs for treating asthma. However, the complicated mechanism of their interaction is not explained clearly, warranting further investigation of that.

\section{Conclusions}

In summary, coadministration of Bud and EEL had superior effects on attenuating airway remodeling; promoting apoptosis; inhibiting autophagy; reducing the expressions of Bcl-2 and Beclin-1; increasing the expressions of Bax, Caspase-3, cleaved Caspase-3, P53, mTOR, and p-mTOR; and regulating $\mathrm{Bcl}-2 / \mathrm{Bax}$ and LC3-II/LC3-I compared with single-Bud treatment. Our study shows that restoring apoptosis and autophagy_-promoting apoptosis and inhibiting autophagy_might be possible mechanisms of combined extracts of EF and LLF with budesonide on improving airway remodeling in ovalbumin-induced asthma rats, providing some ideas for treating asthma in the future. The molecular mechanisms of interplay between autophagy and apoptosis in asthma need further study.

$\begin{array}{ll}\text { Abbreviations } \\ \text { BALF: } & \text { Bronchoalveolar lavage fluid } \\ \text { Bud: } & \text { Budesonide } \\ \text { Bud\&EEL: } & \text { Coadministration of budesonide with the } \\ & \begin{array}{l}\text { extracts of Epimedii Folium and Ligustri } \\ \text { Lucidi Fructus }\end{array} \\ \text { Caspases: } & \text { Cysteine aspartic proteinases } \\ \text { EF: } & \text { Epimedii Folium } \\ \text { EEL: } & \text { The extracts of Epimedii Folium and Ligustri } \\ \text { GCs: } & \text { Lucidi Fructus } \\ \text { H\&E: } & \text { Glucocorticoids } \\ \text { HPA: } & \text { Hematoxylin and eosin } \\ \text { IL: } & \text { Interleukin } \\ \text { IF: } & \text { Immunofluorescence } \\ \text { IOD: } & \text { Integral optical density } \\ \text { i.p.: } & \text { Intraperitoneal injection } \\ \text { i.g.: } & \text { Gastric perfusion } \\ \text { Inhal.: } & \text { Aerosol inhalation } \\ \text { LLF: } & \text { Ligustri Lucidi Fructus } \\ \text { PAS: } & \text { Periodic acid-Schiff } \\ \text { Pbm: } & \text { Perimeter of basement membrane } \\ \text { p-mTOR: } & \text { Phospho-mTOR } \\ \text { qPCR: } & \text { Quantitative real-time PCR }\end{array}$

SD: $\quad$ Standard deviation

s.c.: $\quad$ Subcutaneous injection

TUNEL: TdT-dUTP nick-end labeling

TCM: Traditional Chinese Medicine

Wat: Thickness of airway wall

Wam: Thickness of airway smooth muscle

WB: Western blotting

$\alpha$-SMA: $\quad$ Alpha smooth muscle actin.

\section{Data Availability}

The data used to support the findings of this study are available from the corresponding author upon request.

\section{Ethical Approval}

Our study was performed according to the international, national, and institutional rules considering animal experiments. The protocol for using animals was approved by Ethics Review Committee for Animal Experimentation of Capital Medical University, with the Ethical Inspection Number AEEI-2015-042.

\section{Conflicts of Interest}

The authors declare no conflicts of interest regarding the publication of this paper.

\section{Authors' Contributions}

RHL contributed to the conception and design of this study. RHL and ZTM performed the rat experiments and drafted the manuscript. XFT, YYG, HW, and PY were mainly involved in the experiments and the index detection. All authors have read and approved the manuscript.

\section{Acknowledgments}

Shizeng Li participated in the conception and design of this study, and Yuheng Chen provided some advice about the manuscript. This study was supported by the Natural Science Foundation of China (Grant no. 81873115) and the studio for inheriting national famous Traditional Chinese Medicine expert (Shizeng Li) of State Administration of Traditional Chinese Medicine. The two funders all took part in inspection of experimental rationality and supervision of experimental progress.

\section{References}

[1] C. A. Bonham, K. C. Patterson, and M. E. Strek, "Asthma outcomes and management during pregnancy," Chest, vol. 153, no. 2, pp. 515-527, 2018.

[2] P. K. Henneberger, "Work-exacerbated asthma," Current Opinion in Allergy and Clinical Immunology, vol. 7, no. 2, pp. 146-151, 2007.

[3] W. C. Webley and D. L. Hahn, "Infection-mediated asthma: etiology, mechanisms and treatment options, with focus on chlamydia pneumoniae and macrolides," Respiratory Research, vol. 18, no. 1, p. 98, 2017. 
[4] H. Fehrenbach, C. Wagner, and M. Wegmann, "Airway remodeling in asthma: what really matters," Cell and Tissue Research, vol. 367, no. 3, pp. 551-569, 2017.

[5] S. Bartel, G. Carraro, F. Alessandrini, S. Krauss-Etschmann, F. L. M. Ricciardolo, and S. Bellusci, "miR-142-3p is associated with aberrant WNT signaling during airway remodeling in asthma," American Journal of Physiology Lung Cellular \& Molecular Physiology, vol. 315, no. 2, pp. 328-333, 2018.

[6] Q. N. Yu, Y. B. Guo, X. Li et al., "ILC2 frequency and activity are inhibited by glucocorticoid treatment via STAT pathway in patients with asthma," Allergy, vol. 73, no. 9, pp. 1860-1870, 2018.

[7] S. R. Del Giacco, A. Bakirtas, E. Bel et al., "Allergy in severe asthma," Allergy, vol. 72, no. 2, pp. 207-220, 2017.

[8] K. F. Chung, S. E. Wenzel, J. L. Brozek et al., "International ERS/ATS guidelines on definition, evaluation and treatment of severe asthma," European Respiratory Journal, vol. 43, no. 2, pp. 343-373, 2016.

[9] H. Jiang, X. Chi, X. Zhang, and J. Wang, "Increased serum VDBP as a risk predictor for steroid resistance in asthma patients," Respiratory Medicine, vol. 114, pp. 111-116, 2016.

[10] K. N. Priftis, A. Papadimitriou, M. B. Anthracopoulos, A. Fretzayas, and G. P. Chrousos, "Endocrine-immune interactions in adrenal function of asthmatic children on inhaled corticosteroids," Neuroimmunomodulation, vol. 16, no. 5, pp. 333-339, 2009.

[11] E. W. Zollner, C. Lombard, U. Galal, S. Hough, E. Irusen, and E. Weinberg, "Hypothalamic-pituitary-adrenal axis suppression in asthmatic children on inhaled and nasal corticosteroids-more common than expected?" Journal of Pediatric Endocrinology \& Metabolism, vol. 24, no. 7-8, pp. 529-534, 2011.

[12] M. Sauler, I. S. Bazan, and P. J. Lee, "Cell death in the lung: the apoptosis-necroptosis axis," Annual Review of Physiology, vol. 81, no. 1, pp. 375-402, 2019.

[13] S. Elmore, "Apoptosis: a review of programmed cell death," Toxicologic Pathology, vol. 35, no. 4, pp. 495-516, 2007.

[14] L. Cohen, E. Xueping, J. Tarsi et al., "Epithelial cell proliferation contributes to airway remodeling in severe asthma," American Journal of Respiratory and Critical Care Medicine, vol. 176, no. 2, pp. 138-145, 2007.

[15] C. D. Lucas, D. A. Dorward, S. Sharma et al., "Wogonin induces eosinophil apoptosis and attenuates allergic airway inflammation," American Journal of Respiratory and Critical Care Medicine, vol. 191, no. 6, pp. 626-636, 2015.

[16] P. Ilmarinen and H. Kankaanranta, "Eosinophil apoptosis as a therapeutic target in allergic asthma," Basic \& Clinical Pharmacology \& Toxicology, vol. 114, no. 1, pp. 109-117, 2014.

[17] W. Busse, G. Chupp, H. Nagase et al., "Anti-IL-5 treatments in patients with severe asthma by blood eosinophil thresholds: indirect treatment comparison," Journal of Allergy and Clinical Immunology, vol. 143, no. 1, pp. 190-200, 2019.

[18] T. Y. Jang, C.-S. Park, M.-S. Jeon, M.-J. Heo, K. Na, and Y. H. Kim, "Experimental immunology additive anti-allergic effects of anti-interleukin-33 and anti-siglec-F treatments in a murine model of allergic asthma," Central European Journal of Immunology, vol. 4, no. 4, pp. 426-433, 2014.

[19] K. R. Parzych and D. J. Klionsky, "An overview of autophagy: morphology, mechanism, and regulation," Antioxidants \& Redox Signaling, vol. 20, no. 3, pp. 460-473, 2014.

[20] A. A. Zeki, B. Yeganeh, N. J. Kenyon, M. Post, and S. Ghavami, "Autophagy in airway diseases: a new frontier in human asthma?” Allergy, vol. 71, no. 1, pp. 5-14, 2016.
[21] F. Xia, C. Deng, Y. Jiang et al., "IL4 (interleukin 4) induces autophagy in B cells leading to exacerbated asthma," Autophagy, vol. 14, no. 3, pp. 450-464, 2018.

[22] G.-Y. Ban, D. L. Pham, T. H. K. Trinh et al., "Autophagy mechanisms in sputum and peripheral blood cells of patients with severe asthma: a new therapeutic target," Clinical \& Experimental Allergy, vol. 46, no. 1, pp. 48-59, 2016.

[23] L. J. Martin, J. Gupta, S. S. Jyothula et al., "Functional variant in the autophagy-related 5 gene promotor is associated with childhood asthma," PLoS One, vol. 7, no. 4, Article ID e33454, 2012.

[24] A. C. Racanelli, S. A. Kikkers, A. M. K. Choi, and S. M. Cloonan, "Autophagy and inflammation in chronic respiratory disease," Autophagy, vol. 14, no. 2, pp. 221-232, 2018.

[25] A. Fernández, R. Ordóñez, R. J. Reiter, J. González-Gallego, and J. L. Mauriz, "Melatonin and endoplasmic reticulum stress: relation to autophagy and apoptosis," Journal of Pineal Research, vol. 59, no. 3, pp. 292-307, 2015.

[26] B. E. Fitzwalter and A. Thorburn, "FOXO3 links autophagy to apoptosis," Autophagy, vol. 14, no. 8, pp. 1467-1468, 2018.

[27] V. Nikoletopoulou, M. Markaki, K. Palikaras, and N. Tavernarakis, "Crosstalk between apoptosis, necrosis and autophagy," Biochimica et Biophysica Acta (BBA) - Molecular Cell Research, vol. 1833, no. 12, pp. 3448-3459, 2013.

[28] C. Brosseau, M. Durand, L. Colas et al., "CD9(+) regulatory $\mathrm{B}$ cells induce T cell apoptosis via IL-10 and are reduced in severe asthmatic patients," Frontiers in Immunology, vol. 9, Article ID 3034, 2018.

[29] J. Lv, W. Su, Q. Yu et al., "Heme oxygenase-1 protects airway epithelium against apoptosis by targeting the proinflammatory NLRP3-RXR axis in asthma," Journal of Biological Chemistry, vol. 293, no. 48, pp. 18454-18465, 2018.

[30] R. Liu, X. Kang, L. Xu et al., "Effect of the combined extracts of herba epimedii and fructus ligustri lucidi on sex hormone functional levels in osteoporosis rats," Evidence-based Complementary and Alternative Medicine, vol. 2015, Article ID 184802, 13 pages, 2015.

[31] Y. Q. Tang, C. Li, X. J. Sun et al., "Fructus ligustri lucidi modulates estrogen receptor expression with no uterotrophic effect in ovariectomized rats," BMC Complementary \& Alternative Medicine, vol. 18, no. 1, p. 118, 2018.

[32] S. C. W. Sze, Y. Tong, T. B. Ng, C. L. Y. Cheng, and H. P. Cheung, "Herba epimedii: anti-oxidative properties and its medical implications," Molecules, vol. 15, no. 11, pp. 7861-7870, 2010.

[33] J. Cai, Y. Tian, R. Lin, X. Chen, Z. Liu, and J. Xie, "Protective effects of kidney-tonifying Chinese herbal preparation on substantia nigra neurons in a mouse model of Parkinson's disease," Neural Regeneration Research, vol. 7, no. 6, pp. 413-420, 2012.

[34] Z. T. Mo, W. N. Li, Y. R. Zhai, and Q. H. Gong, "Icariin attenuates $\mathrm{OGD} / \mathrm{R}$-induced autophagy via Bcl-2-dependent cross talk between apoptosis and autophagy in PC12 cells," Evidence Based Complementary \& Alternative Medicine, vol. 2016, Article ID 4343084, 6 pages, 2016.

[35] P. Zhang, H. Li, D. Chen, J. Ni, Y. Kang, and S. Wang, "Oleanolic acid induces apoptosis in human leukemia cells through caspase activation and poly(ADP-ribose) polymerase cleavage," Acta Biochimica et Biophysica Sinica, vol. 39, no. 10, pp. 803-809, 2007.

[36] Y. Song, P. Zhang, Y. Sun et al., "AMPK activation-dependent autophagy compromises oleanolic acid-induced cytotoxicity 
in human bladder cancer cells," Oncotarget, vol. 8, no. 40, pp. 67942-67954, 2017.

[37] R. H. Liu, L. P. Xu, X. Wang, J. Yang, P. Wang, and L. Pan, "Experimental study on effect of combination of epimedii folium/ligustri lucidi fructus and dexamethasone on asthmatic rats," China Journal of Chinese Materia Medica, vol. 37, no. 10, pp. 1497-1499, 2012.

[38] X. Tang, H. Nian, X. Li et al., "Effects of the combined extracts of herba epimedii and fructus ligustri lucidi on airway remodeling in the asthmatic rats with the treatment of budesonide," BMC Complementary and Alternative Medicine, vol. 17, no. 1, p. 380, 2017.

[39] X. Tang, X. Li, H. Nian et al., "Active ingredients of epimedii folium and ligustri lucidi fructus balanced GR/HSP90 to improve the sensitivity of asthmatic rats to budesonide," Evidence Based Complementary \& Alternative Medicine, vol. 2017, Article ID 7961231, 16 pages, 2017.

[40] R. H. Liu, X. Kang, L. P. Xu et al., "Effects of the combined extracts of herba epimedii and fructus ligustri lucidi on bone mineral content and bone turnover in osteoporotic rats," BMC Complementary and Alternative Medicine, vol. 15, p. 112, 2015.

[41] Y. Chen, X. Li, X. Tang et al., "Combined extracts of herba epimedii and fructus ligustri lucidi rebalance bone remodeling in ovariectomized rats," Evidence Based Complementary \& Alternative Medicine, vol. 2019, Article ID 1596951, 11 pages, 2019.

[42] G. An, X. Zhang, W. Wang et al., "The effects of interleukin-33 on airways collagen deposition and matrix metalloproteinase expression in a murine surrogate of asthma," Immunology, vol. 154, no. 4, pp. 637-650, 2018.

[43] Y. Ma, W. Huang, C. Liu et al., "Immunization against TGF$\beta 1$ reduces collagen deposition but increases sustained inflammation in a murine asthma model," Human Vaccines and Immunotherapeutics, vol. 12, no. 7, pp. 1876-1885, 2016.

[44] N. S. Ambhore, R. Katragadda, R. S. Raju Kalidhindi et al., "Estrogen receptor beta signaling inhibits PDGF induced human airway smooth muscle proliferation," Molecular and Cellular Endocrinology, vol. 476, pp. 37-47, 2018.

[45] X.-Z. Guo, X. D. Shao, M. P. Liu et al., "Effect of bax, bcl-2 and bcl-xL on regulating apoptosis in tissues of normal liver and hepatocellular carcinoma," World Journal of Gastroenterology, vol. 8, no. 6, pp. 1059-1062, 2002.

[46] K. Liu, T. Ren, Y. Huang et al., "Apatinib promotes autophagy and apoptosis through VEGFR2/STAT3/BCL-2 signaling in osteosarcoma," Cell Death and Disease, vol. 8, no. 8, Article ID e3015, 2017.

[47] J. Banuelos, S. Shin, Y. Cao et al., "BCL-2 protects human and mouse Th17 cells from glucocorticoid-induced apoptosis," Allergy, vol. 71, no. 5, pp. 640-650, 2016.

[48] Y. N. Wang, L. L. Zhang, X. Y. Fan, S. S. Wu, and S. Q. Zhang, "Poly-L-arginine induces apoptosis of NCI-H292 cells via ERK1/2 signaling pathway," Journal of Immunology Research, vol. 2018, Article ID 3651743, 9 pages, 2018.

[49] C. Rogers, T. Fernandes-Alnemri, L. Mayes, D. Alnemri, G. Cingolani, and E. S. Alnemri, "Cleavage of DFNA5 by caspase-3 during apoptosis mediates progression to secondary necrotic/pyroptotic cell death," Nature Communications, vol. 8, Article ID 14128, 2017.

[50] Y. S. Prakash, C. M. Pabelick, and G. C. Sieck, "Mitochondrial dysfunction in airway disease," Chest, vol. 152, no. 3, pp. 618-626, 2017.

[51] O. Ham, S. Y. Lee, C. Y. Lee et al., "let-7b suppresses apoptosis and autophagy of human mesenchymal stem cells transplanted into ischemia/reperfusion injured heart 7 by targeting caspase3," Stem Cell Research \& Therapy, vol. 6, no. 1, p. 147, 2015.

[52] N. Man, Y. Tan, X.-J. Sun et al., "Caspase-3 controls AML1ETO-driven leukemogenesis via autophagy modulation in a ULK1-dependent manner," Blood, vol. 129, no. 20, pp. 2782-2792, 2017.

[53] R. Huang and W. Liu, "Identifying an essential role of nuclear LC3 for autophagy," Autophagy, vol. 11, no. 5, pp. 852-853, 2015.

[54] E. Jacquin, S. Leclerc-Mercier, C. Judon, E. Blanchard, S. Fraitag, and O. Florey, "Pharmacological modulators of autophagy activate a parallel noncanonical pathway driving unconventional LC3 lipidation," Autophagy, vol. 13, no. 5, pp. 854-867, 2017.

[55] R. Johnson, S. Shabalala, J. Louw, A. P. Kappo, and C. J. F. Muller, "Aspalathin reverts doxorubicin-induced cardiotoxicity through increased autophagy and decreased expression of $\mathrm{p} 53 / \mathrm{mTOR} / \mathrm{p} 62$ signaling," Molecules, vol. 22, no. 10, Article ID 1589, 2017.

[56] G. Liu, F. Pei, F. Yang et al., "Role of autophagy and apoptosis in non-small-cell lung cancer," International Journal of Molecular Sciences, vol. 18, no. 2, Article ID 367, 2017.

[57] J. G. Elliot, P. B. Noble, T. Mauad et al., "Inflammationdependent and independent airway remodelling in asthma," Respirology, vol. 23, no. 12, pp. 1138-1145, 2018.

[58] X. Zhu, Q. Li, G. Hu et al., "BMS-345541 inhibits airway inflammation and epithelial-mesenchymal transition in airway remodeling of asthmatic mice," International Journal of Molecular Medicine, vol. 42, no. 4, pp. 1998-2008, 2018.

[59] Y. Lin, J. Yao, M. Wu et al., "Tetrandrine ameliorates airway remodeling of chronic asthma by interfering TGF- $\beta 1 / \mathrm{Nrf}-2 /$ HO-1 signaling pathway-mediated oxidative stress," Canadian Respiratory Journal, vol. 2019, Article ID 7930396, 12 pages, 2019.

[60] J. Hu, J. Han, and B. Tang, "Expression of interleukin-25 in lung tissues of asthmatic mice and its effect on airway remodeling," Chongqing Medical, vol. 47, no. 12, pp. 16671670, 2018.

[61] S. Holm Nielsen, N. Willumsen, D. J. Leeming et al., "Serological assessment of activated fibroblasts by alpha-smooth muscle actin ( $\alpha$-SMA): a noninvasive biomarker of activated fibroblasts in lung disorders," Translational Oncology, vol. 12, no. 2, pp. 368-374, 2019.

[62] S. R. Reeves, K. A. Barrow, T. K. Kolstad et al., "Fibroblast gene expression following asthmatic bronchial epithelial cell conditioning correlates with epithelial donor lung function and exacerbation history," Scientific Reports, vol. 8, no. 1, Article ID 15768, 2018.

[63] S. Cuylen, C. Blaukopf, A. Z. Politi et al., "Ki-67 acts as a biological surfactant to disperse mitotic chromosomes," Nature, vol. 535, no. 7611, pp. 308-312, 2016.

[64] X. Sun and P. D. Kaufman, "Ki-67: more than a proliferation marker," Chromosoma, vol. 127, no. 2, pp. 175-186, 2018.

[65] R. Rathore, J. E. McCallum, E. Varghese, A.-M. Florea, and D. Büsselberg, "Overcoming chemotherapy drug resistance by targeting inhibitors of apoptosis proteins (IAPs)," Apoptosis, vol. 22, no. 7, pp. 898-919, 2017.

[66] R. S. Wong, "Apoptosis in cancer: from pathogenesis to treatment," Journal of Experimental \& Clinical Cancer Research, vol. 30, no. 1, p. 87, 2011.

[67] A. A. Jarrah, M. Schwarskopf, E. R. Wang et al., "SDF-1 induces TNF-mediated apoptosis in cardiac myocytes," Apoptosis, vol. 23, no. 1, pp. 79-91, 2018.

[68] T. Zhao, Y. Fu, H. Sun, and X. Liu, "Ligustrazine suppresses neuron apoptosis via the $\mathrm{Bax} / \mathrm{Bcl}-2$ and caspase- 3 pathway in 
PC12 cells and in rats with vascular dementia," IUBMB Life, vol. 70, no. 1, pp. 60-70, 2018.

[69] R. Wang, F. Song, S. Li, B. Wu, Y. Gu, and Y. Yuan, "Salvianolic acid A attenuates $\mathrm{CCl} 4$-induced liver fibrosis by regulating the $\mathrm{PI} 3 \mathrm{~K} / \mathrm{AKT} / \mathrm{mTOR}, \mathrm{Bcl}-2 / \mathrm{Bax}$ and caspase-3/ cleaved caspase-3 signaling pathways," Drug Design Development and Therapy, vol. 13, pp. 1889-1900, 2019.

[70] C. Yao, X. Cao, Z. Fu et al., "Boschniakia rossica polysaccharide triggers laryngeal carcinoma cell apoptosis by regulating expression of Bcl-2, caspase-3, and P53," Medical Science Monitor, vol. 23, pp. 2059-2064, 2017.

[71] F. Xing, Q. Zhan, Y. He, J. Cui, S. He, and G. Wang, " $1800 \mathrm{MHz}$ microwave induces p53 and p53-mediated caspase- 3 activation leading to cell apoptosis in vitro," PLoS One, vol. 11, no. 9, Article ID e0163935, 2016.

[72] J. Chen, "The cell-cycle arrest and apoptotic functions of p53 in tumor initiation and progression," Cold Spring Harbor Perspectives in Medicine, vol. 6, no. 3, Article ID a026104, 2016.

[73] X. Wang, E. R. Simpson, and K. A. Brown, "p53: protection against tumor growth beyond effects on cell cycle and apoptosis," Cancer Research, vol. 75, no. 23, pp. 5001-5007, 2015.

[74] A. Kondratskyi, K. Kondratska, R. Skryma, D. J. Klionsky, and N. Prevarskaya, "Ion channels in the regulation of autophagy," Autophagy, vol. 14, no. 1, pp. 3-21, 2018.

[75] I. H. Cho, Y. J. Choi, J. H. Gong, D. Shin, M. K. Kang, and Y. H. Kang, "Astragalin inhibits autophagy-associated airway epithelial fibrosis," Respiratory Research, vol. 16, no. 1, p. 51, 2015.

[76] J. M. M. Levy, C. G. Towers, and A. Thorburn, “Targeting autophagy in cancer," Nature Reviews Cancer, vol. 17, no. 9, pp. 528-542, 2017.

[77] A. F. Corona Velazquez and W. T. Jackson, "So many roads: the multifaceted regulation of autophagy induction," Molecular \& Cellular Biology, vol. 38, no. 21, 2018.

[78] S. R. Yoshii and N. Mizushima, "Monitoring and measuring autophagy," International Journal of Molecular Sciences, vol. 18, no. 9, 2017.

[79] K. Wang, "Autophagy and apoptosis in liver injury," Cell Cycle, vol. 14, no. 11, pp. 1631-1642, 2015.

[80] Y. Chung, J. Lee, S. Jung, Y. Lee, J. W. Cho, and Y. J. Oh, "Dysregulated autophagy contributes to caspase-dependent neuronal apoptosis," Cell Death \& Disease, vol. 9, no. 12, p. 1189, 2018.

[81] L. Ou, S. Lin, B. Song, J. Liu, R. Lai, and L. Shao, "The mechanisms of graphene-based materials-induced programmed cell death: a review of apoptosis, autophagy, and programmed necrosis," International Journal of Nanomedicine, vol. 12, pp. 6633-6646, 2017. 